\title{
SheAR VISCOSITY CALCULATION FOR PARTICLE-BASED FLOW
}

\author{
by \\ Pradeep Kunwar \\ Master of Probability and Statistics, Tribhuvan University, 2002 \\ Bachelor of Science, Tribhuvan University, 1998
}

\author{
A thesis \\ presented to Ryerson University \\ in partial fulfillment of the \\ requirements for the degree of \\ Master of Science \\ in the Program of \\ Applied Mathematics
}

Toronto, Ontario, Canada, 2014

(C)Pradeep Kunwar 2014 


\section{Author's Declaration}

I hereby declare that I am the sole author of this thesis. This is a true copy of the thesis, including any required final revisions, as accepted by my examiners.

I authorize Ryerson University to lend this thesis to other institutions or individuals for the purpose of scholarly research.

I further authorize Ryerson University to reproduce this thesis by photocopying or by other means, in total or in part, at the request of other institutions or individuals for the purpose of scholarly research.

I understand that my thesis may be made electronically available to the public. 


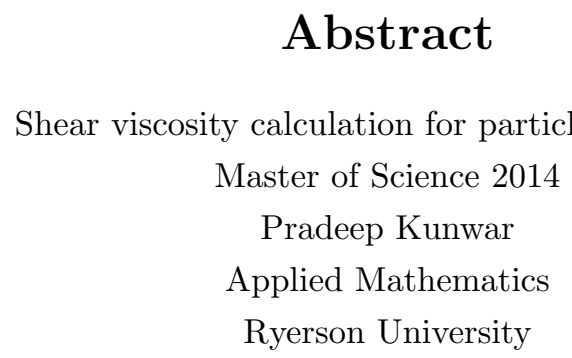

A particle-based method called multi-particle collision (MPC) dynamics is considered, and the shear viscosity is calculated theoretically. As part of the particle-based mechanism, velocities of particles change due to collisions and due to an applied external force used to create flow. The system's temperature increases due to the external force, and a thermostat is used to remove this excess temperature so as to maintain constant temperature (isothermal) flow conditions. A theoretical expression for the shear viscosity is derived and compared to existing viscosity expressions.

Additionally, results for MPC flow through a local constriction are assessed. The novelty of the numerical results in this Thesis come from using a local thermostat rather than a global thermostat that had been used in the past. 


\section{Acknowledgements}

It is a great pleasure to me to express a few words about the people who contributed and supported me for making this thesis possible. First of all, my sincere thanks goes to my academic parents. My supervisor Dr.Katrin Rohlf supervised my thesis and provided inexorable support. She gave me her valuable time and any help even though she was very busy. So, I would like to thank her heartily. I would like to thank Dr. Pascal, Dr. Ilie Dr. Lan, and Dr. Pablo who gave me academic support. Secondly, my thanks also goes to those friends who supported me. Hom Kandel and Bhai Kumar Adhikari who helped me directly and indirectly. They gave me some ideas related to this work and courses. Thirdly, I would like to thank Steve Kanellis, who helped me technically. He helped me many times. When I faced problems with my laptop, he solved my problems with Miktex errors and data backup immediately. Finally, I would like to extend my thanks to my family. My mother and brothers and sisters inspired me about education learning. 


\section{Dedication}

I would like to dedicate this thesis to my father who is not now. He always inspired and motivated me to learn mathematics and statistics. 


\section{Contents}

1 Introduction $\quad 1$

2 MPC Background $\quad 6$

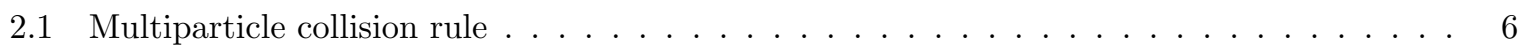

2.2 Force and Thermostat $\ldots \ldots \ldots \ldots \ldots \ldots \ldots$

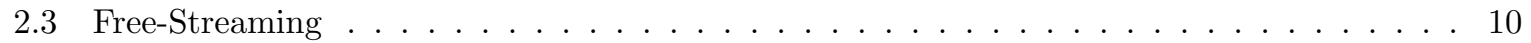

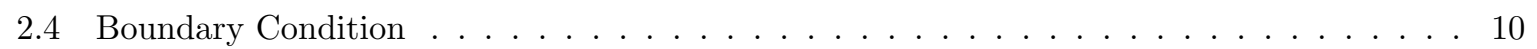

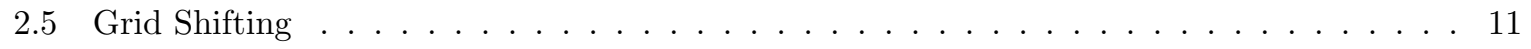

2.6 Averaging Procedure $(\mathrm{MPC}) \ldots \ldots \ldots \ldots \ldots \ldots \ldots \ldots$

2.7 Theoretical Averages . . . . . . . . . . . . . . . . . . . . . . 13

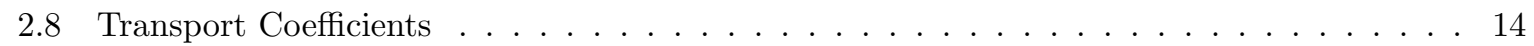

3 Viscosity Calculation $\quad \mathbf{1 5}$

3.1 Velocities in MPC Dynamics . . . . . . . . . . . . . . . . . . . 15

3.2 Viscosity for Large Mean Free Path . . . . . . . . . . . . . . . . . . . . . 16

4 Numerical Results $\quad 23$

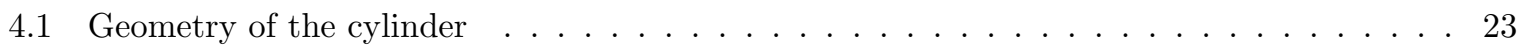

4.2 Cell Energy Generation . . . . . . . . . . . . . . . . . . . . . 24

4.2 .1 Acceptance Rejection Method . . . . . . . . . . . . . . . . . . 25

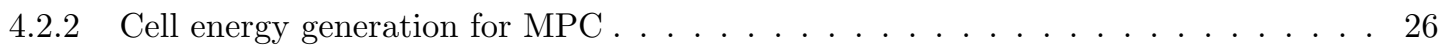

4.3 Numerical Results . . . . . . . . . . . . . . . . . . . . . . . . . . . . . 29

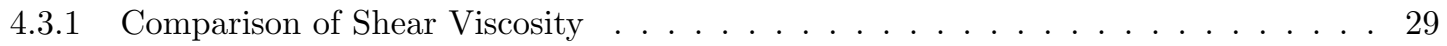

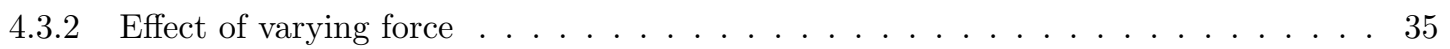

4.3 .3 Effect of varying degree of constriction . . . . . . . . . . . . . 35

4.3 .4 Effect of varying slip . . . . . . . . . . . . . . . . . . . . . 44

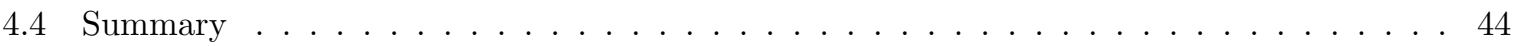

5 Conclusions and Future Work $\quad 47$

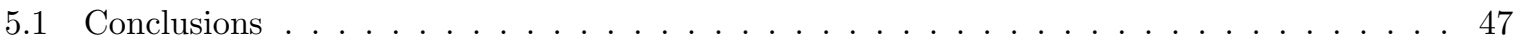

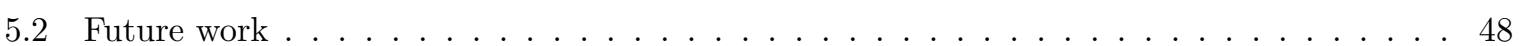


References 


\section{List of Tables}

4.1 Comparison of theoretically predicted viscosities to the numerically obtained value, using viscosity expressions with and without thermostat. . . . . . . . . . . 35 


\section{List of Figures}

2.1 Diagrammatic representation of the multiparticle collision rule. The upper panel shows the center of mass velocity and pre-collision velocities of the particles relative to the center of mass. The lower panel shows the result of adding back the center of mass velocities to get the post-collision velocities. The figure is modified from $[36] . \ldots \ldots \ldots$

2.2 Top figure shows MPC-BB $(\lambda=0)$ and bottom figure shows MPC-LIT $(\lambda=0.5) . \ldots 12$

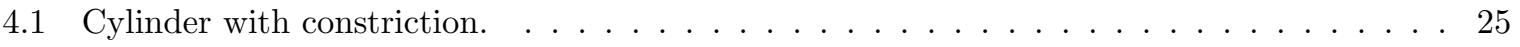

4.2 Target probability density function $p(E)$ compared to the bounding probability density

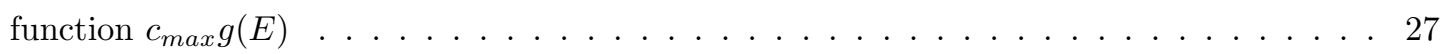

4.3 Comparison of the histogram of 10,000 random numbers generated using the Acceptance Rejection method to the target probability density curve $p(E) \ldots \ldots \ldots \ldots$

4.4 Comparison of shear viscosity with and without thermostat. . . . . . . . . . . . 30

4.5 Axial velocity cross-section for MPC-BB (no-slip) with best parabolic fit. The equation for the parabolic fit is $v=-0.2885539209+0.0825548278 y-0.002845674 y^{2}$. . . . . . . 31

4.6 Axial velocity cross-section for MPC-LIT (slip) with best parabolic fit. The equation for the parabolic fit is $v=-0.258305808+0.084189849 y-0.002904962 y^{2} \ldots \ldots \ldots \ldots$. . . 32

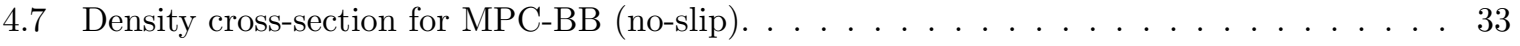

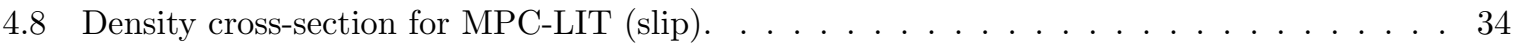

4.9 Scaled centerline densities for various values of $g$ in a constriction with $\delta=0.5$ in the

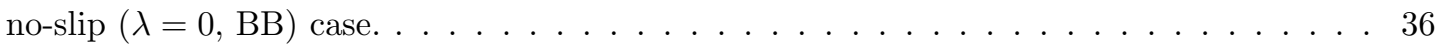

4.10 Scaled centerline densities for various values of $g$ in a constriction with $\delta=0.5$ and slip

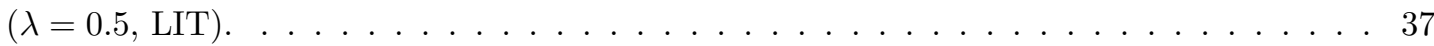

4.11 Scaled centerline velocities for various values of $g$ in a constriction with $\delta=0.5$ in the

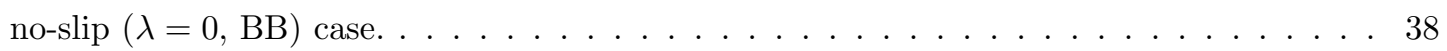

4.12 Scaled centerline velocities for various values of $g$ in a constriction with $\delta=0.5$ and slip $(\lambda=0.5, \mathrm{LIT}) \ldots \ldots \ldots \ldots \ldots \ldots \ldots$

4.13 Scaled centerline densities for various values of $\delta$ in a constriction with $g=0.005$ in the

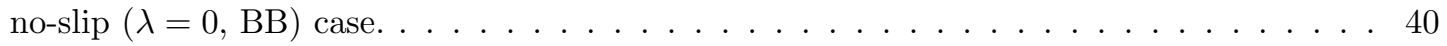

4.14 Scaled centerline densities for various values of $\delta$ in a constriction with $g=0.005$ and slip

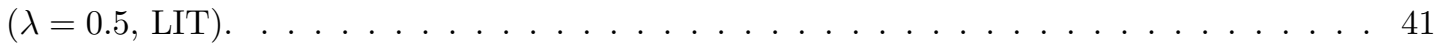


4.15 Scaled centerline velocities for various values of $\delta$ in a constriction with $g=0.005$ in the

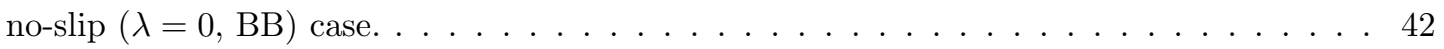

4.16 Scaled centerline velocities for various values of $\delta$ in a constriction with $g=0.005$ and slip

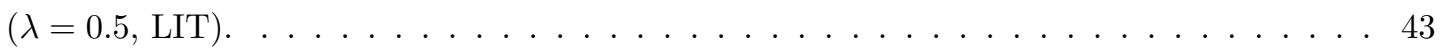

4.17 Scaled centerline densities for various values of $\lambda$ in a constriction with $g=0.005$ and

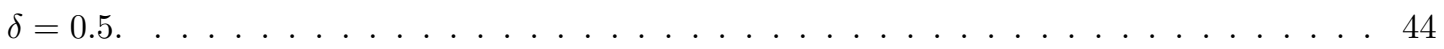

4.18 Scaled centerline velocities for various values of $\lambda$ in a constriction with $g=0.005$ and

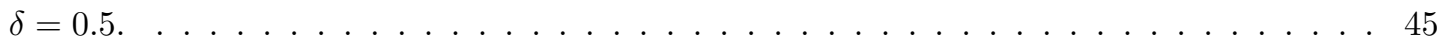




\section{List of Appendices}

Appendix A 


\section{Chapter 1}

\section{Introduction}

The blood circulatory system of the human body is very complex and delicate, consisting of the heart, blood and blood vessels, each with its own distinct function. The heart is also known as the blood-pump which pumps $70 \mathrm{ml}$ of blood through the circulatory system with each heart beat. Blood is a bio-fluid consisting of plasma and cells, that delivers nutrients and oxygen to the body cells and removes waste products of metabolism from those cells. In general, blood cells consist of red blood cells, white blood cells and platelets. Approximately 7 percent of the total body weight in the human body is blood, and the density of blood is approximately $1060 \mathrm{~kg} / \mathrm{m}^{3}$, very close to that of water $\left(1000 \mathrm{~kg} / \mathrm{m}^{3}\right)$. Blood vessels are elastic, tubular, circulatory channels, such as an artery, a vein, or a capillary, through which the blood circulates from the heart to the cells and from the cells to the heart. A viscoelastic model has even been used to investigate the deformation of vessels when blood flows, and an arterial network model was created for a branch of the blood circulation in the body consisting of a network of 45 viscoelastic branches [4].

For effective circulation, each organ in the system should remain in healthy condition and should carry out its functions in a cyclic fashion. Any interruption in the system can affect human health and survival directly. Among all the circulatory problems, blood flow problems are primarily determined by the blood vessel's patency although there are several other contributing factors. Narrowing, or stenosis, of blood vessels is the primary cause of impaired circulation, which typically results from a condition called atherosclerosis. Atherosclerosis is the thickening and narrowing of blood vessels as a result of accumulation of calcium and fatty materials such as cholesterol and triglycerides. This limits the flow of oxygen-rich blood to the target organ of the body and stops it from functioning properly. In the 2005 database of the World Health Organization (WHO), 52 percent of the deaths of people in Hungary were caused by circulatory disease. Nowadays, circulatory disease is increasing significantly in many countries [4].

The exact cause of atherosclerosis is unknown. Many researchers believe it begins with an injury to the innermost layer of the artery known as the endothelium. Researchers believe that factors such as high blood pressure, elevated LDL (low density lypo-protein, or bad cholesterol), smoking, diabetes, inflammation, obesity, an unhealthy diet, lack of exercise and family history of heart disease can con- 
tribute to the damage. Once the artery is damaged, blood cells called platelets build up there to try to heal the injury. Over time, fats, cholesterol, and other substances also build up at the site, which thickens and hardens the artery wall. The blood flow through the artery is decreased, and the oxygen supply to organs also decreases. Blood clots may also form, potentially blocking the artery, or entering the bloodstream and cutting off blood supply to other organs.

The effects of atherosclerosis differ depending upon which arteries in the body are affected, but it primarily causes coronary artery disease such as angina (chest pain on exertion) and a heart attack or myocardial infarction, cerebrovascular disease such as stroke, with the potential for permanent brain damage, and peripheral artery disease such as narrowing in the arteries of the legs. Poor circulation results in leg pain and poor wound healing, leading to amputations and renal disease. The axial velocity, shear stress distribution, and resistance to flow are flow variables that have been determined for different diseases such as polycythemia, plasma cell dyscrasias and Hb SS (sickle cell), and normal blood. Results show that values of shear stress increase with an increase in the degree of stenosis, and decrease with an increase of couple stress. A difference in axial velocities, shear stresses and resistance to flow were found for different diseases and compared to normal blood [49].

There are many important studies related to hemorheology that have been performed in biomedical research. The key parameters in physiology that play an important role in blood flow are: blood pressure, lumen diameter, whole blood viscosity, compliance of vessels, and peripheral vascular resistance. Some parameters in hemorheology, such as whole blood viscosity, plasma viscosity, hematocrit, RBC deformability and aggregation, and fibrinogen concentration in plasma also play an important role in arteriosclerotic vascular disease. Much research has been conducted to determine the blood viscosity in patients with diseases such as ischemic heart disease and myocardial infarction. In [29], it was found that there was a correlation between plasma viscosity and disease. Other researchers tried to find the relationship between hemorheological parameters and blood flow. Rheological characteristics of human blood plasma play an important role in the flow of blood [10].

In general, a fluid is a substance that has no shape of its own. The shape of a fluid depends on the container it is in. Water, milk, blood, ethanol and air are fluids. Water, glycerine, air and ethanol are examples of a Newtonian fluid. These types of fluids exhibit Newtonian characteristics for the shearstress shear-rate relationship. A Newtonian shear-stress shear-rate plot is a straight line whose positive slope is the viscosity of the fluid.

If the graph between shear stress and shear rate is not a straight line, the fluid is classified as nonNewtonian. There are many non-Newtonian models such as the power law model, the modified power law model, and the Cross model for example. Another class of non-Newtonian models are viscoelastic models. Blood is often treated as a non-Newtonian viscoelastic fluid. Other commonly used models for blood include the Bingham plastic model, the Herschel-Bulkley model, the Casson model, and the Quemada model.

Scientists and researchers in bio-medical fields are constantly looking for new approaches and they often use modern computers to investigate and explore. Numerical simulation is a technique often used to model the real world with the help of computer programming, that can be used to study the flow behavior of a complex fluid. Simulation for complex fluids is a very challenging task for researchers 
because of large resolution requirements, and diverse spatial and temporal scales. There are two major methods used to visualize complex flow behavior. In general, these methods can be classified as the Eulerian grid method, and the Lagrangian particle method.

Complex fluids have complex flow properties and are typically systems with large components that are not easily describable using microscopic molecular dynamic simulation methods, because of large system size and the inability to control the variables and the technological setting for study. Many of these phenomena depend on such things as molecular weight, molecular number, and molecular shape just to name a few. That is why researchers often choose mesoscopic methods. In mesoscopic physics, the size of a particle is typically between $10^{-6}$ to $10^{-9} \mathrm{~m}$. If the size of the particle is between $10^{-12}$ and $10^{-9} \mathrm{~m}$, or less than $10^{-12} \mathrm{~m}$, it is generally treated using microscopic and quantum physics approaches respectively. In this Thesis, the mesoscopic approach is used.

Discrete particle-based simulations in the field of fluid dynamics are basically divided into two groups namely lattice-based simulation methods, and off-lattice based simulation methods. The Boltzmann method and lattice gas automata are the most popular simulation methods in lattice-based simulation methods. The Boltzmann method is a powerful technique that can be used to simulate complex fluid flow in complex flow geometries. It is based on the Boltzmann equation, and particles reside on specified grid points with discrete velocities based on the grid configuration. In this method, fluid particles "collide" at discrete time steps. Lattice gas automata, or lattice gas cellular automata, are based on cellular automata for simulation of fluid flow. The basic principle of the cellular automaton was invented by Stanislaw Ulam and John von Neumann in 1940. Lattices consist of a discrete number of grid points in each cell. The grid can be in any finite number of dimensions. At the beginning, cells are assigned a particular state, and updated according to some mathematical rule that prescribes their next state, and so on.

The most important off-lattice particle-based methods in fluid dynamics are dissipative particle dynamics (DPD), direct simulation Monte-Carlo methods (DSMC) and multiparticle collision dynamics (MPC). DPD was initially introduced by Hoogerbrugge and Koelman in 1992 [24]. DPD is a combination of molecular dynamics and Langevin dynamics. In DPD, each particle is considered as a group of molecules or atoms, and positions and velocities of each particle are considered as continuous variables. Particle interactions are due to three types of forces namely conservative forces, dissipative forces and random forces. Conservative forces are used to model soft repulsion. Time steps are large and the system rapidly reaches equilibrium. Dissipative forces are used for friction forces that reduce the relative velocity of the particles. Random forces are used to compensate for loss of kinetic energy due to dissipative forces. In this case, Newton's second law can be written as a sum of these three forces, although the conservative forces and the dissipative forces are negative because conservative forces are repulsive and dissipative forces are friction forces (opposite force of system). The DSMC method is a particle-based technique for simulation of fluid flow, appropriate for low density fluids. It was first proposed by Bird [7]. Here, stochastic methods are used to solve the non-linear, time-dependent Boltzmann equations. In the DSMC system, positions and velocities of the particle are continuous variables. The state of the system is thus defined by the positions and velocities of the particles. The geometry under consideration is divided into small cubical cells called collision cells, that provides a coarse-grained model for the system. In 
general, there are two main steps and these steps are free-streaming, and collisions. In free-streaming, if there is no interaction between particles and no contact with the wall, particles move freely, and the locations of the particles are updated according to the previous location and the current velocity of the particle. In the collision step, it is assumed that particles collide with each other randomly, so the particles are selected randomly within a cell for the collision. From the kinetic theory principles, the collision probability for hard spheres is directly proportional to their relative velocity. In a cell, particle $i$ and $j$ are two randomly selected particles that collide with each other. If the absolute relative velocity of these particles is greater than the product of a random number $R$ chosen from the uniform distribution on $[0,1)$ with the local maximum relative speed, namely if

$$
\left|\mathbf{v}_{i}-\mathbf{v}_{j}\right|>R \mathbf{v}_{\max }
$$

then a collision occurs. For finding the collision probability, only the magnitude of the velocities of the particles is used and particles moving away from one another can also collide with this rule. Conservation of momentum and conservation of energy provide four out of six equations that can be used to determine the post-collision velocities of the particles. For the remaining two equations, it is assumed that the direction of the relative post-collision velocities is uniformly distributed on the unit sphere. This means that the remaining two conditions lead to a randomly chosen direction.

Multiparticle collision (MPC) dynamics was first introduced by Malevanets and Kapral in 1990 and is a method that conserves energy and momentum in the system [36]. Most of the concepts between MPC and DSMC are similar, with the key difference between them being the collision step. In the collision step, post-collision velocities of particles in a collision cell are obtained using a rotation angle $\alpha$ or $-\alpha$. Therefore, the rotational operator, which is a stochastic rotation matrix, is used. This concept is not part of the DSMC method, and that is one of the main reasons why MPC collisions are more efficient numerically compared to DSMC collisions. Nowadays, MPC has become very popular in fluid dynamics applications and has also been used in many other areas. Lamura and Gompper applied multiparticle collision dynamics to simulate flow around a square and a circular cylinder in 2 dimensions for Reynolds numbers ranging from 10 to 130 [34]. Allahyarov and Gompper investigated multiparticle collision dynamics in three dimensional solvent flows in a channel with and without a spherical obstacle [3]. Undulatory locomotion, the type of motion characterized by wave-like movement patterns, of fishlike shapes moving through a fluid have been investigated in two dimensional multiparticle collision dynamics [51]. Bedkihal et. al. have used MPC to study the steady flow through a constricted cylinder [6]. One of the most important contributions to MPC dynamics is provided by Bolintineanu et. al. [9]. They provided an in-depth discussion of the parameters of MPC dynamics such as, slip and no-slip at the wall, cell size, viscosity, mean free path and consequences for fluid solid boundaries and thermostats.

Thermostatting is a process that automatically adjusts the temperature in a system to a desired level in a particle-based method through velocity rescaling. The most common thermostats are the Anderson thermostat, the Berendsen thermostat, the Nose-Hoover thermostat, and the Langevin (stochastic) thermostat. MPC with the Anderson thermostat has been introduced by Allahyarov and Gompper [3]. 
In this method the velocity in the collision step is prescribed as

$$
\mathbf{v}_{i(n e w)}=\mathbf{v}_{\xi}^{G}+\mathbf{v}_{i}^{\text {ran }}-\frac{\sum_{j \in c e l l} \mathbf{v}_{j}^{\text {ran }}}{M_{\xi}}
$$

where $\mathbf{v}_{i}^{r a n}$ is a velocity chosen randomly from the Maxwell Boltzmann velocity distribution, $M_{\xi}$ is the number of particles in the $\xi^{t h}$ cell, and $\mathbf{v}_{\xi}^{G}$ is the mean velocity in the particular cell. Note that $i$ corresponds to particle $i$, and $\mathbf{v}_{i(n e w)}$ denotes the velocity of particle $i$ after the collision step. This scheme is denoted by MPC-AT and it can be shown that it conserves linear momentum and kinetic energy, but not angular momentum. To additionally incorporate angular momentum, a modified MPC-AT was introduced. This method was denoted by MPC-AT+a [42, 21]. Later, the Anderson Thermostat was introduced by Koopmana and C. P. Lowe in 2006 [33]. They compared the Anderson thermostat with the Lower Anderson Thermostat and claimed that the Lower Anderson Thermostat was significantly more efficient and more convenient than the previous Anderson Thermostat. The Lower Anderson Thermostat conserved momentum and was Galilean invariant. In [23], a thermostat was used to interact with the system at different time rates. Strong Thermostatting Interaction and Partial thermostatting interaction were applied in MPC dynamics. A strong interaction was obtained by applying the thermostat at every simulation step and weaker interaction was achieved by applying the thermostat at larger time intervals.

The specific aim of this Thesis is to study the dynamic fluids of flows of an MPC fluid in a cylinder with a local constriction using a force applied along the $y$-axis to create flow in the system. Particle velocities are changed due to collisions, flow acceleration and thermostatting. Collisions in MPC dynamics, as well as free-streaming of the particles conserve mass, momentum and energy. Applying a force in the $y$ direction adds energy to the system, changing the system temperature. To keep the system temperature fixed, a thermostat is used to rescale the velocities. As part of our main contribution, the shear viscosity is explicitly calculated theoretically, and discussed in the context of numerical results for flow through cylinders with a local constriction.

This thesis is organized as follows: In chapter 2 the MPC background is provided discussing the collision rule, the forcing, the thermostat, the connection between averages of the MPC system with macroscopic flow variables, and equilibrium probability distributions. In chapter 3 , the explicit expression for viscosity is calculated for the MPC dynamics described in chapter 2. Chapter 4 provides some further details regarding the numerical implementation of the MPC dynamics, as well as simulation results for flow through a local constriction. In the last chapter 5 , we conclude the thesis with some important conclusions and future work. 


\section{Chapter 2}

\section{MPC Background}

An MPC fluid consists of $N$ identical point particles in a system volume that is divided into cubic cells. The mass of each particle is $m$, and particles are uniformly distributed in the system. $L_{x}, L_{y}$ and $L_{z}$ are the number of cells in the $x, y$ and $z$ directions respectively. On average, each cell $\xi$ contains $M$ particles. Each particle has a continuous position $\mathbf{r}_{i}$ and velocity $\mathbf{v}_{i},(i=1,2,3, \ldots, N)$ and these are updated at discrete time intervals $\tau$. A phase space is formed by particle positions and momenta and is denoted by $\boldsymbol{\Pi}=\left(\mathbf{r}_{i}^{N}, \mathbf{P}_{i}^{N}\right)=\left(\mathbf{r}_{1}, m \mathbf{v}_{1}, \ldots . ., \mathbf{r}_{N}, m \mathbf{v}_{N}\right)$. In the system, there are $N$ particles and each particle has three momentum components and three position coordinates. Therefore the dimension of phase space is $6 N$. Particles undergo a sequence of steps at each time interval. First, particles collide with the MPC collision rule, next, particles are accelerated by the external force in the $y$-direction, and velocities are rescaled through a thermostat to maintain constant temperature in the system. Next, particles free-stream.

In this Chapter, we outline the details of the key steps in the MPC dynamics: collisions, application of a force with subsequent thermostatting, and free-streaming. Next we discuss in Section 2.4 the dynamics imposed on particles when they hit the cylinder boundary. In Section 2.5 we discuss the gridshifting mechanism that is also invoked at each time step so as to ensure Galilean invariance in the MPC dynamics. In Section 2.6 we describe the averaging mechanism used in order to connect the mescoscopic system to the macroscopic flow velocities. Next, Section 2.7 provides the equilibrium distributions that will be used when determining the theoretical expressions for the macroscopic viscosity of the MPC flow. For flows that are not subjected to acceleration and thermostatting, the viscosity is presented in Section 2.8 .

\subsection{Multiparticle collision rule}

According to the multiparticle collision rule, the pre-collision velocity vector $\mathbf{v}_{i}^{\prime}$ of particle $i, \quad(i=$ $1,2,3, \ldots, N)$ is rotated randomly by means of a rotation operator $\mathbf{W}_{\xi}$, relative to the average cell velocity (center of mass velocity) $\mathbf{V}_{\xi}^{\prime}$. The post-collision velocity $\mathbf{v}_{i}^{\prime \prime}$ of the $i^{t h}$ particle becomes 


$$
\mathbf{v}_{i}^{\prime \prime}=\mathbf{V}_{\xi}^{\prime}+\mathbf{W}_{\xi}\left(\mathbf{v}_{i}^{\prime}-\mathbf{V}_{\xi}^{\prime}\right)
$$

where

$$
\mathbf{V}_{\xi}^{\prime}=\frac{\sum_{i=1}^{M} \mathbf{v}_{i}^{\prime}}{M}
$$

and $\mathrm{M}$ is the average number of particles in a cell. The rotation operator is defined as

$$
\mathbf{W}_{\xi}=\left(\begin{array}{ccc}
R_{11} & R_{12} & R_{13} \\
R_{21} & R_{22} & R_{23} \\
R_{31} & R_{32} & R_{33}
\end{array}\right)=\left(\begin{array}{ccc}
l_{x}^{2}+\left(1-l_{x}^{2}\right) c & l_{x} l_{y}(1-c)-l_{z} s & l_{x} l_{z}(1-c)+l_{y} s \\
l_{x} l_{y}(1-c)+l_{z} s & l_{y}^{2}+\left(1-l_{y}^{2}\right) c & l_{y} l_{z}(1-c)-l_{x} s \\
l_{x} l_{z}(1-c)-l_{y} s & l_{y} l_{z}(1-c)+l_{x} s & l_{z}^{2}+\left(1-l_{z}^{2}\right) c
\end{array}\right)
$$

where $l_{x}=\sqrt{1-\theta^{2}} \cos (\varphi), l_{y}=\sqrt{1-\theta^{2}} \sin (\varphi), l_{z}=\theta, c=\cos (\alpha), s=\sin (\alpha)$, and $\varphi$ and $\theta$ are uncorrelated random variables chosen from a uniform distribution on the interval $[0,2 \pi]$ and $[-1,1]$ respectively, and $\alpha$ is a random rotation angle often chosen to be $\pm \pi / 2$.

Momentum and energy are conserved locally in a collision cell by the collision rule (2.1). Suppose $m$ is the mass of the particles and $M$ is the number of particles in cell $\xi$. The momenta before and after the collision of particles are $\sum_{i=1}^{M} m \mathbf{v}_{i}^{\prime}$ and $\sum_{i=1}^{M} m \mathbf{v}_{i}^{\prime \prime}$ respectively. Similarly, the kinetic energy before and after the collision are $\frac{\sum_{i=1}^{M} m\left|\mathbf{v}_{i}^{\prime}\right|^{2}}{2}$ and $\frac{\sum_{i=1}^{M} m\left|\mathbf{v}_{i}^{\prime \prime}\right|^{2}}{2}$ respectively. It has been shown in [1] that $\sum_{i=1}^{M} m \mathbf{v}_{i}^{\prime}=\sum_{i=1}^{M} m \mathbf{v}_{i}^{\prime \prime}$ and $\frac{\sum_{i=1}^{M} m\left|\mathbf{v}_{i}^{\prime}\right|^{2}}{2}=\frac{\sum_{i=1}^{M} m\left|\mathbf{v}_{i}^{\prime \prime}\right|^{2}}{2}$.

In Figure 2.1, we show a diagrammatic representation of a collision for two particles even though the rule works for any number of particles in a given collision cell. Note that the collisions in MPC dynamics are not the sum of pairwise collisions as used in DSMC, and hence are much more efficient numerically. Using the notation $\mathbf{v}_{i}^{\prime \prime}=\left(\begin{array}{c}v_{i x}^{\prime \prime} \\ v_{i y}^{\prime \prime} \\ v_{i z}^{\prime \prime}\end{array}\right), \mathbf{V}_{\xi}^{\prime}=\left(\begin{array}{c}V_{\xi x}^{\prime} \\ V_{\xi y}^{\prime} \\ V_{\xi z}^{\prime}\end{array}\right), \mathbf{v}_{i}^{\prime}=\left(\begin{array}{c}v_{i x}^{\prime} \\ v_{i y}^{\prime} \\ v_{i z}^{\prime}\end{array}\right)$, we can rewrite the explicit velocity components of $\mathbf{v}_{i}^{\prime \prime}$ according to (2.1) - (2.3) as:

$$
\begin{aligned}
& v_{i x}^{\prime \prime}=V_{\xi x}^{\prime}+c\left[\left(v_{i x}^{\prime}-V_{\xi x}^{\prime}\right)-\sum_{\beta=x, y, z}\left(v_{i \beta}^{\prime}-V_{\xi \beta}^{\prime}\right) l_{\beta} l_{x}\right]+s\left[-\left(v_{i y}^{\prime}-V_{\xi y}^{\prime}\right) l_{z}+\left(v_{i z}^{\prime}-V_{\xi z}^{\prime}\right) l_{y}\right]+\sum_{\beta=x, y, z}\left(v_{i \beta}^{\prime}-V_{\xi \beta}^{\prime}\right) l_{\beta} l_{x}, \\
& v_{i y}^{\prime \prime}=V_{\xi y}^{\prime}+c\left[\left(v_{i y}^{\prime}-V_{\xi y}^{\prime}\right)-\sum_{\beta=x, y, z}\left(v_{i \beta}^{\prime}-V_{\xi \beta}^{\prime}\right) l_{\beta} l_{y}\right]-s\left[\left(v_{i y}^{\prime}-V_{\xi y}^{\prime}\right) l_{z}-\left(v_{i x}^{\prime}-V_{\xi x}^{\prime}\right) l_{x}\right]+\sum_{\beta=x, y, z}\left(v_{i \beta}^{\prime}-V_{\xi \beta}^{\prime}\right) l_{\beta} l_{y},
\end{aligned}
$$

and

$$
v_{i z}^{\prime \prime}=V_{\xi z}^{\prime}+c\left[\left(v_{i z}^{\prime}-V_{\xi z}^{\prime}\right)-\sum_{\beta=x, y, z}\left(v_{i \beta}^{\prime}-V_{\xi \beta}^{\prime}\right) l_{\beta} l_{z}\right]+s\left[\left(v_{i y}^{\prime}-V_{\xi y}^{\prime}\right) l_{x}+\left(v_{i x}^{\prime}-V_{\xi x}^{\prime}\right) l_{y}\right]+\sum_{\beta=x, y, z}\left(v_{i \beta}^{\prime}-V_{\xi \beta}^{\prime}\right) l_{\beta} l_{z}
$$



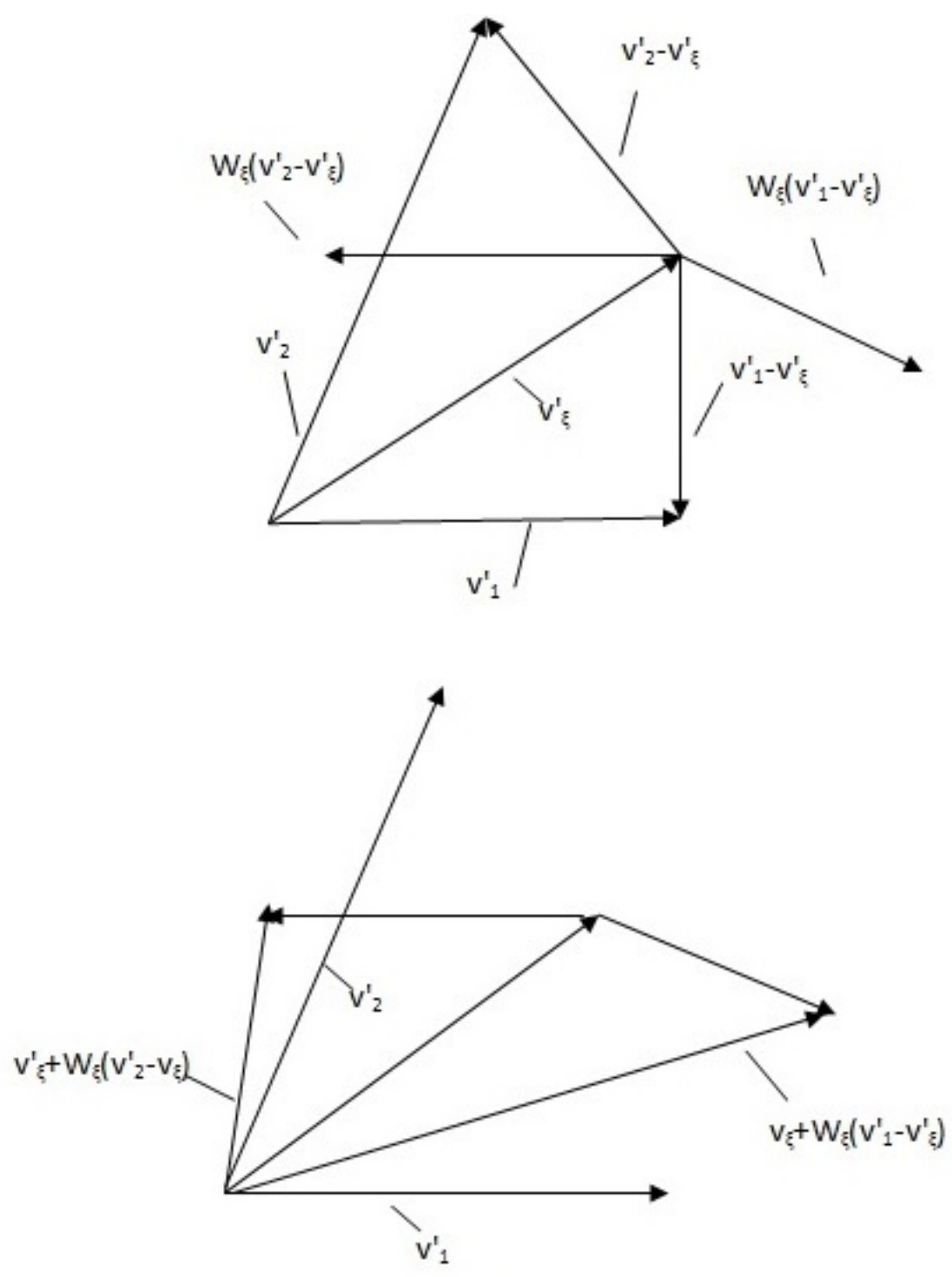

Figure 2.1: Diagrammatic representation of the multiparticle collision rule. The upper panel shows the center of mass velocity and pre-collision velocities of the particles relative to the center of mass. The lower panel shows the result of adding back the center of mass velocities to get the post-collision velocities. The figure is modified from [36]. 
The expressions for $v_{i x}^{\prime \prime}$ and $v_{i z}^{\prime \prime}$ can also be found in Tüzel [56], and are discussed in more detail in Section 3.1 in the next chapter.

\section{$2.2 \quad$ Force and Thermostat}

To achieve flow, a force $\mathbf{F}=m \mathbf{g}=m\left(\begin{array}{l}0 \\ g \\ 0\end{array}\right)$ is applied to all particles in the system. The force creates flow in the $y$-direction. The post-collision velocities $\mathbf{v}_{i}^{\prime \prime}$ of particles are updated according to the following rule

$$
\mathbf{v}_{i}^{\prime \prime \prime}=\mathbf{v}_{i}^{\prime \prime}+\frac{\mathbf{F}}{m} \tau=\mathbf{v}_{i}^{\prime \prime}+g \tau\left(\begin{array}{l}
0 \\
1 \\
0
\end{array}\right)
$$

where $\tau$ is the discrete time step.

The system energy increases if an external force is applied to the system this way, as the force accelerates the fluid particles. Due to this increase, the actual temperature $T^{\prime \prime}$ of the system also increases. To create isothermal flow with constant temperature $T$, a scaling factor $h$ is introduced, that can be used to maintain the local temperature. This is called thermostatting. Incorporating the local thermostat, gives the thermostat rule $\mathbf{v}_{i}=\mathbf{V}_{\xi}^{\prime \prime \prime}+$ rescaling due to the thermostat, or rather

$$
\mathbf{v}_{i}=\mathbf{V}_{\xi}^{\prime \prime \prime}+h\left(\mathbf{v}_{i}^{\prime \prime \prime}-\mathbf{V}_{\xi}^{\prime \prime \prime}\right),
$$

where

$$
h=\sqrt{\frac{2 E_{\xi}}{m \sum_{i=1}^{M}\left\|\mathbf{v}_{i}^{\prime \prime \prime}-\mathbf{V}_{\xi}^{\prime \prime \prime}\right\|^{2}}}=\sqrt{\frac{2 E_{\xi}}{m \sum_{i=1}^{M}\left\|\mathbf{v}_{i}^{\prime}-\mathbf{V}_{\xi}^{\prime}\right\|^{2}}},
$$

and $E_{\xi}$ is a random number drawn from the Gamma Distribution (see section 2.7), that varies from cell to cell and in time.

In order to see that the denominator for $h$ in (2.9) in terms of triple-primed variables is the same is that in terms of primed variables, we note that

$$
\begin{aligned}
\left\|\mathbf{v}_{i}^{\prime \prime \prime}-\mathbf{V}_{\xi}^{\prime \prime \prime}\right\|^{2} & =\left(\mathbf{v}_{i}^{\prime \prime \prime}-\mathbf{V}_{\xi}^{\prime \prime \prime}\right)^{T}\left(\mathbf{v}_{i}^{\prime \prime \prime}-\mathbf{V}_{\xi}^{\prime \prime \prime}\right) \\
& =\left(\mathbf{V}_{\xi}^{\prime}+\mathbf{W}_{\xi}\left(\mathbf{v}_{i}^{\prime}-\mathbf{V}_{\xi}^{\prime}\right)+\mathbf{g} \tau-\mathbf{g} \tau-\mathbf{V}_{\xi}^{\prime}\right)^{T}\left(\mathbf{V}_{\xi}^{\prime}+\mathbf{W}_{\xi}\left(\mathbf{v}_{i}^{\prime}-\mathbf{V}_{\xi}^{\prime}\right)+\mathbf{g} \tau-\mathbf{g} \tau-\mathbf{V}_{\xi}^{\prime}\right) \\
& =\left(\mathbf{W}_{\xi}\left(\mathbf{v}_{i}^{\prime}-\mathbf{V}_{\xi}^{\prime}\right)\right)^{T}\left(\mathbf{W}_{\xi}\left(\mathbf{v}_{i}^{\prime}-\mathbf{V}_{\xi}^{\prime}\right)\right) \\
& =\left(\mathbf{v}_{i}^{\prime}-\mathbf{V}_{\xi}^{\prime}\right)^{T} \mathbf{W}_{\xi}^{T} \mathbf{W}_{\xi}\left(\mathbf{v}_{i}^{\prime}-\mathbf{V}_{\xi}^{\prime}\right) \\
& =\left\|\mathbf{v}_{i}^{\prime}-\mathbf{V}_{\xi}^{\prime}\right\|^{2},
\end{aligned}
$$


where from $(2.3)$

$$
\begin{aligned}
\mathbf{W}_{\xi}^{T} \mathbf{W}_{\xi} & =\left(\begin{array}{lll}
R_{11} & R_{12} & R_{13} \\
R_{21} & R_{22} & R_{23} \\
R_{31} & R_{32} & R_{33}
\end{array}\right)^{T}\left(\begin{array}{lll}
R_{11} & R_{12} & R_{13} \\
R_{21} & R_{22} & R_{23} \\
R_{31} & R_{32} & R_{33}
\end{array}\right) \\
& =\left(\begin{array}{lll}
c_{11} & c_{12} & c_{13} \\
c_{21} & c_{22} & c_{23} \\
c_{31} & c_{32} & c_{33}
\end{array}\right),
\end{aligned}
$$

with $c_{i i}=l_{x}^{2}+l_{y}^{2}+l_{z}^{2}=1$, for $i=1,2,3$ and $c_{i j}=0, i \neq j$, for $i, j=1,2,3$. Thus

$$
\mathbf{W}_{\xi}^{T} \mathbf{W}_{\xi}=\mathbf{I}_{3 \times 3} .
$$

\subsection{Free-Streaming}

Since there are continuous positions $\mathbf{r}_{i}^{\prime}$ and velocities $\mathbf{v}_{i}$, free-streaming updates the positions of particles in a time step $\tau$ according to

$$
\mathbf{r}_{i}=\mathbf{r}_{i}^{\prime}+\mathbf{v}_{i} \tau
$$

The notation used here is consisted with the previous sections in that $\mathbf{r}_{i}^{\prime}$ is the position of the $i^{\text {th }}$ particle at the beginning of the time step, and $\mathbf{v}_{i}$ is the velocity after the collision, acceleration and thermostat steps have occurred.

\subsection{Boundary Condition}

If an obstacle is placed in the system around which the fluid has to flow, the flow is affected by the obstacle. Therefore the presence of an interface severely affects the dynamics of fluid flows. At the macroscopic level, when fluids come in contact with a solid phase, the normal component of the velocity at the interface must be zero. This means that it is impossible for the fluid to pass through the boundary. The tangential velocity inherently depends upon the interaction at the interface. There are two ideal situations in terms of boundary conditions, namely no-slip (or stick) and slip. In case of no-slip, the tangential velocity of the fluid relative to that of the boundary must be zero. In slip boundary conditions, the tangential component of the fluid is non-zero.

There are several ways to implement these boundary conditions at the mesoscopic level. The more common no-slip condition is generally implemented by means of a bounce-back (BB) rule. Malevanets and Kapral [36] used the BB boundary condition when they introduced the MPC dynamics. When a particle collides with a boundary, the normal and tangential components of velocities of particles are reversed in BB. In this case the average relative velocity of the fluid near the wall is zero because the relative velocity distribution of particles reflected from the wall mirrors the distribution of particles approaching the wall [61]. In blood flow applications, the endothelium in blood vessels may give rise to 
some slip. In this case, a generalized mesoscopic boundary condition that can incorporate slip at the wall is needed. Such a BC was introduced in [61] and can be written mathematically as

$$
\begin{gathered}
\mathbf{v}_{n}=-\mathbf{v}_{n}^{\prime}, \\
\mathbf{v}_{t}=(2 \lambda-1) \mathbf{v}_{t}^{\prime} .
\end{gathered}
$$

Here, $\mathbf{v}_{n}^{\prime}$ represents the normal component of the velocity of a particle before collision with the wall and $\mathbf{v}_{n}$ represents the normal component of the velocity of the particle after collision with the wall. Similarly $\mathbf{v}_{t}^{\prime}$ represents the tangential component of the velocity before hitting the wall and $\mathbf{v}_{t}$ represents the tangential component of the velocity after hitting the wall. The $(2 \lambda-1)$ factor determines the degree of slip, and its value can be varied.

When $\lambda=0$, then $\mathbf{v}_{t}=-\mathbf{v}_{t}^{\prime}$, giving the BB condition that leads to macroscopic no-slip.

When $\lambda=0.5$, then $\mathbf{v}_{t}=0$, giving a loss-in-tangential (LIT) condition with large macroscopic slip.

When $\lambda=1$, then $\mathbf{v}_{t}=\mathbf{v}_{t}^{\prime}$, giving rise to a perfect slip condition that leads to uniform flow.

Unless $\lambda=0$ or 1 , this boundary condition results in some energy of the particle being lost when it hits the wall. Therefore energy is not conserved in most cases incorporating slip this way. This effect is minimized since a thermostat is used in our simulations.

\subsection{Grid Shifting}

Mean free path is the average distance traveled by a moving particle between collisions with other moving particles. If the mean free path is less than the cell size, the same particles could collide more than once with each other through the MPC collision rule. Such multiple collisions are also possible near the boundary of the flow domain where velocities are typically smallest. If this happens, Galilean invariance is no longer satisfied, and the molecular chaos assumption is violated (velocities are not uncorrelated and independent). To overcome this problem, a grid shifting mechanism is used. This shifting mechanism was first proposed by Ihle and Kroll [27]. In the grid shifting mechanism, before the collision step takes place, all particles are shifted by the same uniformly distributed random translation vector. The particles' positions are thus randomized by the shift and it has been shown that this method restores Galilean invariance [54].

\subsection{Averaging Procedure (MPC)}

Tysanner and Garcia have explained that calculating mean fluid properties is very easy, but depending on the method used can lead to biased measurement in particle-based simulations of fluid flow [57]. Two commonly used averaging methods are sample average measurement (SAM) and cumulative average measurement (CAM). In SAM the average particle velocity in each cell at each sample is computed first and then averaged over all samples. CAM sums the velocities of all particles in a cell over all the samples, and then divides this cumulative total by the cumulative total number of particles in the cell to get the 

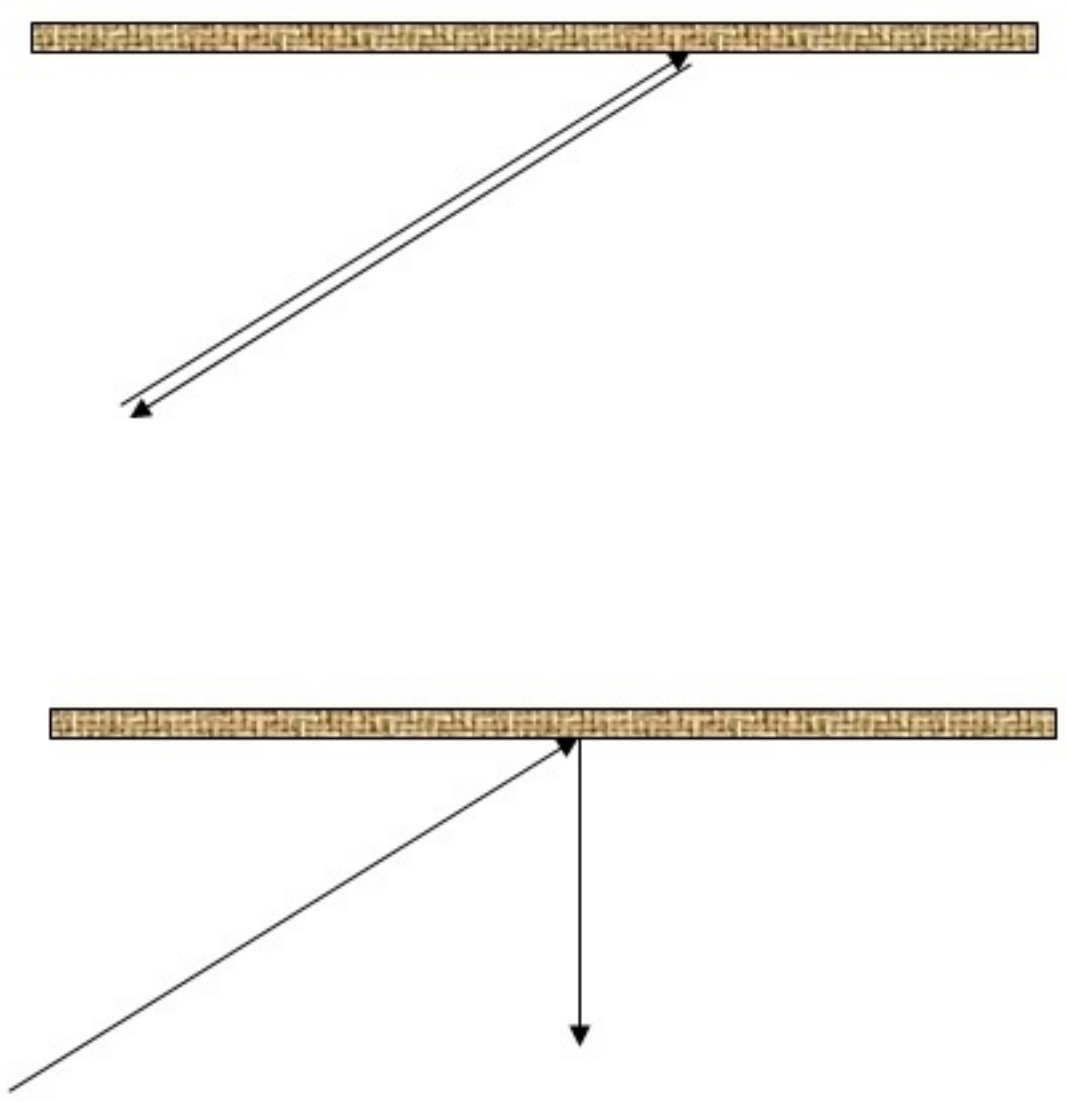

Figure 2.2: Top figure shows MPC-BB $(\lambda=0)$ and bottom figure shows MPC-LIT $(\lambda=0.5)$. 
mean velocity per cell. Tysanner and Garcia have shown that SAM can have bias due to the correlation of fluctuations in the system and that CAM can be used to remove these correlations. Mathematically, CAM can be expressed as,

$$
\left\langle\mathbf{u}_{\xi}\right\rangle=\frac{\sum_{i \in \xi}^{N_{\xi}\left(t_{1}\right)} \mathbf{v}_{i}\left(t_{1}\right)+\sum_{i \in \xi}^{N_{\xi}\left(t_{2}\right)} \mathbf{v}_{i}\left(t_{2}\right)+\ldots \ldots+\sum_{i \in \xi}^{N_{\xi}\left(t_{s}\right)} \mathbf{v}_{i}\left(t_{s}\right)}{N_{\xi}\left(t_{1}\right)+N_{\xi}\left(t_{2}\right)+\ldots \ldots .+N_{\xi}\left(t_{s}\right)}
$$

where, $\left\langle\mathbf{u}_{\xi}\right\rangle$ is the average velocity in cell $\xi$, $\xi$ is the cell number, $i \in \xi$ means that particle $i$ is inside cell $\xi, N_{\xi}\left(t_{j}\right)$ is the number of particles in cell $\xi$ at time $t_{j}, s$ is the number of samples used in the average process and $\left\langle\mathbf{u}_{\xi}\right\rangle$ is the macroscopic velocity vector for particles in the same cell.

Note that it is assumed that the system satisfies the ergodic hypothesis of thermodynamics and instead of using many samples, successive time steps of a very long simulation are used instead. It has been argued that the stochastic nature of MPC dynamics leads to noisy averages [46], but the CAM method overcomes this problem and leads to smooth averages. Note that Malevaents and Kapral used a simple weighted average [36, 37] although this method is not as common.

\subsection{Theoretical Averages}

In computing theoretical averages in the next chapter, the following probability density functions will be used. The velocity distribution of an isothermal MPC flow at equilibrium, has been shown to be the Maxwell-Boltzmann probability distribution. It is given by

$$
p(\{\mathbf{v}\})=\left(\frac{m}{2 \pi k_{B} T}\right)^{\frac{3 N}{2}} \exp \left(-\frac{m}{2 k_{B} T} \sum_{i=1}^{N} \mathbf{v}_{i}^{2}\right)
$$

where $N$ is the total number of particles in the system, $k_{B}$ is the Boltzmann constant, $T$ is temperature of the system, $m$ is the mass of the particles, and $\{\mathbf{v}\}=\mathbf{v}_{1}, \mathbf{v}_{2}, \ldots, \mathbf{v}_{N}$ are the velocities of the particles in the system. This probability distribution will be used in computing averages of velocities so as to connect to macroscopic flow variables.

The probability density function for the kinetic energy $E_{\xi}$ of particles in cell $\xi$ is prescribed to follow the Gamma Distribution with parameters $\phi / 2$ and $k_{B} T$. Mathematically, it is given by

$$
p\left(E_{\xi} \mid N_{\xi}\right)=\frac{1}{E_{\xi} \Gamma\left(\frac{\phi}{2}\right)}\left(\frac{E_{\xi}}{k_{B} T}\right)^{\frac{\phi}{2}} \exp \left(-\frac{E_{\xi}}{k_{B} T}\right) .
$$

Here $p\left(E_{\xi} \mid N_{\xi}\right)$ is the conditional probability density function for energy $E_{\xi}$ in the $\xi^{\text {th }}$ cell given $N_{\xi}$ particles in the cell, $\phi=3\left(N_{\xi}-1\right)$ is the number of degrees of freedom within a collision cell $\xi, \Gamma$ is the Gamma function, $T$ is the temperature of the system, and $k_{B}$ is the Boltzmann constant. This distribution is the assumed distribution for the thermostat used in the MPC dynamics. 


\subsection{Transport Coefficients}

It is possible to calculate viscosity and other macroscopic transport coefficients for MPC dynamics. In the case of no forcing, Noguchi and Gompper [21] have shown that the fluid viscosity $\mu$ is equal to the sum of a kinetic component $\mu_{k i n}$ and a collision part $\mu_{c o l}$. The simple expression, for the $\alpha= \pm \frac{\pi}{2}$ rule used in this Thesis, for 3D flow is given by

$$
\begin{gathered}
\mu_{k i n}=\frac{\rho k_{B} T}{m}\left[\frac{5 n}{6\left(n-1+e^{-n}\right)}-\frac{1}{2}\right] \Delta t \\
\mu_{\text {coll }}=\frac{m}{18 a_{0} \Delta t}\left(n-1+e^{-n}\right) \\
\mu=\mu_{\text {kin }}+\mu_{\text {coll }}
\end{gathered}
$$

where, $\rho=$ mass density $=\frac{m n}{a_{0}^{3}}, n$ is the average number of particles in a cell, $m$ is the mass of a particle and $a_{0}$ is the length of each cubic cell. This viscosity is the theoretically expected value for MPC flow without forcing/thermostatting, and differs from our theoretical expression derived in the next chapter. 


\section{Chapter 3}

\section{Viscosity Calculation}

Using Green-Kubo relations, the viscosity of a fluid can be computed by calculating appropriate averages using velocities of particles in the system, and equilibrium probability distributions. In Section 3.1 we provide the explicit relationship between $\mathbf{v}(t+\tau)$ and $\mathbf{v}(t)$ according to the MPC dynamics described in

Chapter 2. In Section 3.2, we provide the details for approximating the subsequent viscosity of the MPC flow using such Green-Kubo relations. Explicit expressions for the dynamics described in Chapter 2 do not exist in the literature. Expression exist for flow that is not accelerated, and without a thermostat, as provided in section 2.8 , but not for accelerated, thermostatted flow.

\subsection{Velocities in MPC Dynamics}

In Chapter 2, the key steps affecting the velocity $\mathbf{v}_{i}(t)$ of particle $i$ at time $t$ in a given time step are outlined in detail. Notation here is consistent with Chapter 2 and a schematic representation of the dynamics is as follows:

$$
\mathbf{v}_{i}(t)=\mathbf{v}_{i}^{\prime} \stackrel{\text { collision }}{\longrightarrow} \mathbf{v}_{i}^{\prime \prime} \stackrel{\text { force }}{\longrightarrow} \mathbf{v}_{i}^{\prime \prime \prime} \stackrel{\text { thermostat }}{\longrightarrow} \quad \mathbf{v}_{i}=\mathbf{v}_{i}(t+\tau)
$$

The mathematical expressions for each of these steps are given in (2.1), (2.7), and (2.8) and are

$$
\begin{gathered}
\mathbf{v}_{i}^{\prime \prime}=\mathbf{V}_{\xi}^{\prime}+\mathbf{W}_{\xi}\left(\mathbf{v}_{i}^{\prime}-\mathbf{V}_{\xi}^{\prime}\right)(\text { collision}) \\
\mathbf{v}_{i}^{\prime \prime \prime}=\mathbf{v}_{i}^{\prime \prime}+g \tau(0,1,0)^{T}(\text { force }) \\
\mathbf{v}_{i}(t+\tau)=\mathbf{V}_{\xi}^{\prime \prime \prime}+h\left(\mathbf{v}_{i}^{\prime \prime \prime}-\mathbf{V}_{\xi}^{\prime \prime \prime}\right) \text { (thermostat). }
\end{gathered}
$$

Combining these to write $\mathbf{v}_{i}(t+\tau)$ in terms of $\mathbf{v}_{i}(t)$ gives

$$
\mathbf{v}_{i}(t+\tau)=\mathbf{V}_{\xi}(t)+h \mathbf{W}_{\xi}\left(\mathbf{v}_{i}(t)-\mathbf{V}_{\xi}(t)\right)+g \tau(0,1,0)^{T}
$$


Note that

$$
\mathbf{V}_{\xi}^{\prime}(t)=\frac{\sum_{i=1}^{M} \mathbf{v}_{i}^{\prime}}{M}=\frac{\sum_{i=1}^{M} \mathbf{v}_{i}^{\prime \prime}}{M}=\mathbf{V}_{\xi}^{\prime \prime}
$$

and

$$
\mathbf{V}_{\xi}^{\prime \prime \prime}=\frac{\sum_{i=1}^{M} \mathbf{v}_{i}^{\prime \prime \prime}}{M}=\frac{\sum_{i=1}^{M} \mathbf{v}_{i}^{\prime \prime}}{M}+g \tau(0,1,0)^{T}=\mathbf{V}_{\xi}^{\prime \prime}+g \tau(0,1,0)^{T}
$$

Using

$$
\mathbf{W}_{\xi}=\left(\begin{array}{lll}
R_{11} & R_{12} & R_{13} \\
R_{21} & R_{22} & R_{23} \\
R_{31} & R_{32} & R_{33}
\end{array}\right),
$$

from (2.3), the $x, y$, and $z$ components of $\mathbf{v}_{i}(t+\tau)$ are

$$
\begin{gathered}
v_{i x}(t+\tau)=V_{\xi x}(t)+h\left[R_{11}\left(v_{i x}(t)-V_{\xi x}(t)\right)+R_{12}\left(v_{i y}(t)-V_{\xi y}(t)\right)+R_{13}\left(v_{i z}(t)-V_{\xi z}(t)\right)\right] \\
v_{i y}(t+\tau)=V_{\xi y}(t)+g \tau+h\left[R_{21}\left(v_{i x}(t)-V_{\xi x}(t)\right)+R_{22}\left(v_{i y}(t)-V_{\xi y}(t)\right)+R_{23}\left(v_{i z}(t)-V_{\xi z}(t)\right)\right] \\
v_{i z}(t+\tau)=V_{\xi z}(t)+h\left[R_{31}\left(v_{i x}(t)-V_{\xi x}(t)\right)+R_{32}\left(v_{i y}(t)-V_{\xi y}(t)\right)+R_{33}\left(v_{i z}(t)-V_{\xi z}(t)\right)\right] .
\end{gathered}
$$

Comparing (3.8) with (2.3) and using the explicit expressions for $R_{i j}$ for $i, j=1,2,3$ these equations can be written as

$$
\begin{gathered}
v_{i x}(t+\tau)=V_{\xi x}(t)+h\left[c\left(\left(v_{i x}(t)-V_{\xi x}(t)\right)-\sum_{\beta=x, y, z}\left(v_{i \beta}(t)-V_{\xi \beta}(t)\right) l_{\beta} l_{x}\right)\right. \\
\left.\left.-s\left(\left(v_{i y}(t)-V_{\xi y}(t)\right) l_{z}-\left(v_{i z}(t)-V_{\xi z}(t)\right) l_{y}\right)+\sum_{\beta=x, y, z}\left(v_{i \beta}(t)-V_{\xi \beta}(t)\right) l_{\beta} l_{x}\right)\right] \\
\begin{array}{c}
v_{i y}(t+\tau)=V_{\xi y}(t)+g \tau+h\left[c\left(\left(v_{i y}(t)-V_{\xi y}(t)\right)-\sum_{\beta=x, y, z}\left(v_{i \beta}(t)-V_{\xi \beta}(t)\right) l_{\beta} l_{y}\right)\right. \\
\left.\left.-s\left(\left(v_{i y}(t)-V_{\xi y}(t)\right) l_{z}-\left(v_{i x}(t)-V_{\xi x}(t)\right) l_{x}\right)+\sum_{\beta=x, y, z}\left(v_{i \beta}(t)-V_{\xi \beta}(t)\right) l_{\beta} l_{y}\right)\right] \\
v_{i z}(t+\tau)=V_{\xi z}(t)+h\left[c\left(\left(v_{i z}(t)-V_{\xi z}(t)\right)-\sum_{\beta=x, y, z}\left(v_{i \beta}(t)-V_{\xi \beta}(t)\right) l_{\beta} l_{z}\right)\right. \\
\left.\left.-s\left(\left(v_{i y}(t)-V_{\xi y}(t)\right) l_{x}-\left(v_{i x}(t)-V_{\xi x}(t)\right) l_{y}\right)+\sum_{\beta=x, y, z}\left(v_{i \beta}(t)-V_{\xi \beta}(t)\right) l_{\beta} l_{z}\right)\right]
\end{array}
\end{gathered}
$$

\subsection{Viscosity for Large Mean Free Path}

From [56], the shear viscosity $\nu$ for large mean free path can be approximated by

$$
\nu=\frac{\mu}{\rho} \approx \frac{\tau}{N k_{B} T}\left(\frac{C_{0}}{2}+\sum_{n=1}^{\infty} C_{n}\right),
$$


where the first term is taken as half of $C_{0}$ and

$$
C_{n}=\frac{\sum_{i, j}<v_{i x}(0) \Delta \xi_{i z}(0) v_{j x}(n \tau) \Delta \xi_{j z}(n \tau)>}{\tau^{2}}, \text { for } n \geq 0 .
$$

Here, $\Delta \xi_{i z}(t)=\xi_{i z}(t+\tau)-\xi_{i z}(t)$ is the change in the $z$-component of the position of particle $i$ from time $t$ to $t+\tau$, and $\Delta \xi_{i z}(t+n \tau)=\xi_{i z}(t+(n+1) \tau)-\xi_{i z}(t+n \tau)$ is the change in the $z$-component of the position of particle $i$ from time $t+n \tau$ to $t+(n+1) \tau$. The bracket notation $\langle\cdot\rangle$ is used to denote averaging over the equilibrium distribution, namely

$$
<f\left(\mathbf{v}_{i}\right)>=\int_{-\infty}^{\infty} f\left(\mathbf{v}_{i}\right) p(\{\mathbf{v}\}) d\{\mathbf{v}\}
$$

where $p(\{\mathbf{v}\})$ was specified in equation (2.15). In this calculation, we take $m=1$.

From Ihle and Kroll [28], one can approximate $\Delta \xi_{i z}$ with $\tau v_{i z}$ for evaluation of $C_{n}$, but not for $C_{0}$. Thus

$$
C_{n} \approx \sum_{i, j}<v_{i x}(0) v_{i z}(0) v_{j x}(n \tau) v_{j z}(n \tau)>, n>0
$$

In other words [28],

$$
C_{0} \approx \frac{\sum_{i, j}<v_{i x}(0) \Delta \xi_{i z}(0) v_{j x}(0) \Delta \xi_{j z}(0)>}{\tau^{2}}=\frac{N k_{B} T}{\tau^{2}}<\left(\Delta \xi_{z}\right)^{2}>
$$

and

$$
C_{1} \approx \sum_{i, j}<v_{i x}(0) v_{i z}(0) v_{j x}(\tau) v_{j z}(\tau)>
$$

In evaluating $C_{1}$, we note that $C_{1}$ has diagonal contributions $(i=j)$ and off-diagonal contributions $(i \neq j)$.

For the diagonal contributions of $C_{1}$, using (3.9) - (3.11), we can write

$$
\begin{aligned}
<v_{i x}(0) & v_{i z}(0) v_{i x}(\tau) v_{i z}(\tau)>=<R_{11} R_{33}><h^{2}\left[v_{i x}^{2}(0) v_{i z}^{2}(0)-v_{i x}^{2}(0) v_{i z}(0) V_{\xi z}(0)-v_{i z}^{2}(0) v_{i x}(0) V_{\xi x}(0)\right. \\
& \left.+v_{i x}(0) v_{i z}(0) V_{\xi x}(0) V_{\xi z}(0)\right]>+<R_{13} R_{31}><h^{2}\left[v_{i x}^{2}(0) v_{i z}^{2}(0)-v_{i x}^{2}(0) v_{i z}(0) V_{\xi z}(0)-v_{i z}^{2}(0) v_{i x}(0) V_{\xi x}(0)\right. \\
& \left.+v_{i x}(0) v_{i z}(0) V_{\xi x}(0) V_{\xi z}(0)\right]>+<R_{11}><h\left[v_{i x}^{2}(0) v_{i z}(0) V_{\xi z}(0)-v_{i x}(0) v_{i z}(0) V_{\xi x}(0) V_{\xi z}(0)\right]> \\
& +<R_{33}><h\left[v_{i z}^{2}(0) v_{i x}(0) V_{\xi x}(0)-v_{i x}(0) v_{i z}(0) V_{\xi x}(0) V_{\xi z}(0)\right]>+<v_{i x}(0) v_{i z}(0) V_{\xi x}(0) V_{\xi z}(0)>.
\end{aligned}
$$

Recall the definition of the $R_{i j}$ from (2.3), and note that the bracket notation for the $R_{i j}$ indicates averages over $\theta$ and $\varphi$, namely

$$
4 \pi<R_{i j}>=\int_{0}^{2 \pi} \int_{-1}^{1} R_{i j} d \theta d \varphi, \quad i, j=1,2,3 .
$$


After manually computing all averages, we have

$$
\left[<\mathbf{R}_{i j}>\right]=\left(\begin{array}{ccc}
<R_{11}> & <R_{21}> & <R_{31}> \\
<R_{12}> & <R_{22}> & <R_{32}> \\
<R_{13}> & <R_{23}> & <R_{33}>
\end{array}\right)=\left(\frac{1}{3}+\frac{2 c}{3}\right) \mathbf{I}_{3 X 3}
$$

where $\mathbf{I}_{3 X 3}$ is the $3 X 3$ identity matrix.

Similarly,

$$
4 \pi<R_{i j} R_{k l}>=\int_{0}^{2 \pi} \int_{-1}^{1} R_{i j} R_{k l} d \theta d \varphi, \quad i, j=1,2,3 \text { and } k, l=1,2,3 .
$$

Performing all calculations, the symmetric matrix of $\left\langle R_{i j} R_{k l}\right\rangle$ is 


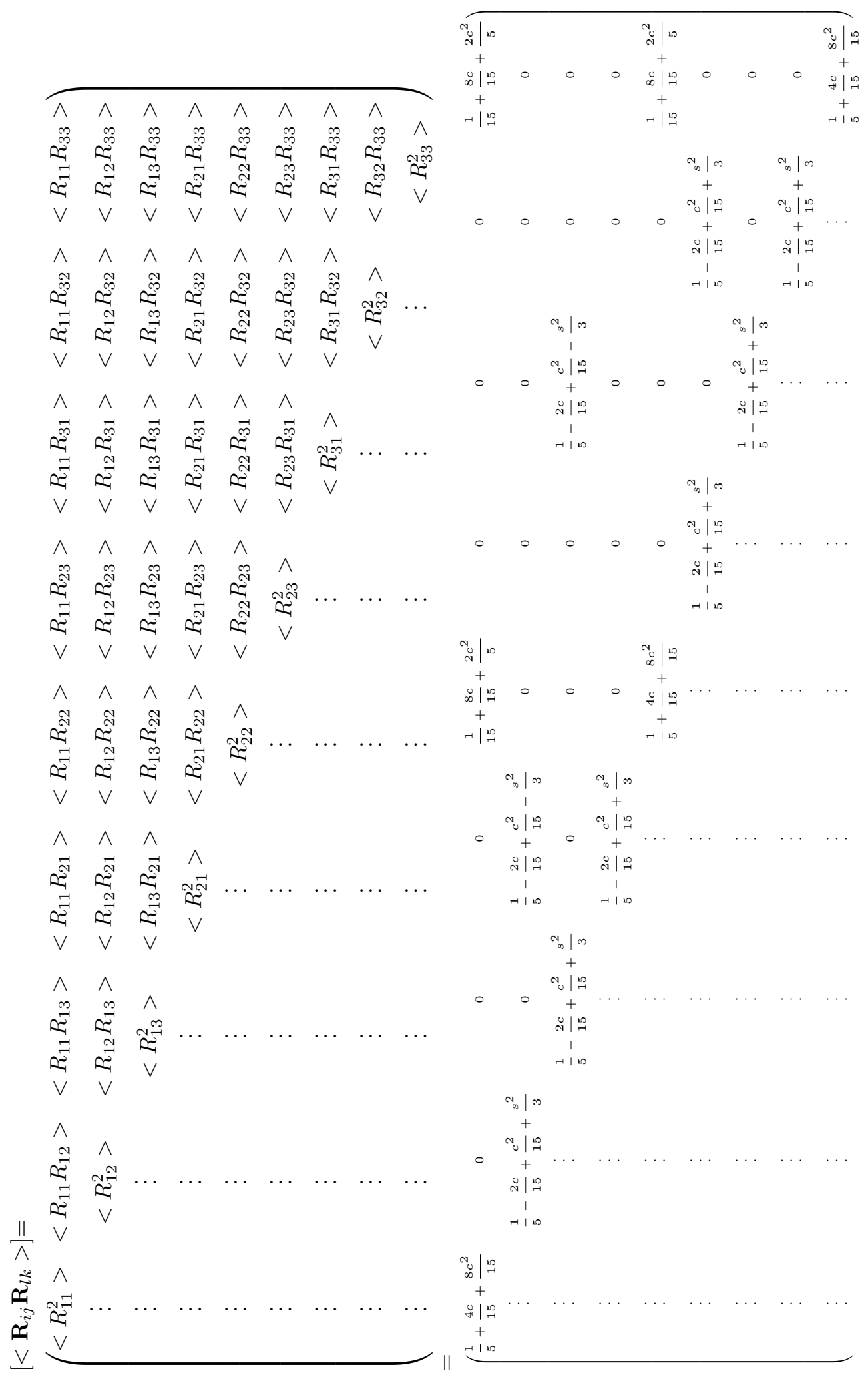


Substituting these values for $<R_{i j}>$ and $<R_{i j} R_{k l}>$ into (3.18) gives

$$
\begin{aligned}
<v_{i x}(0) & v_{i z}(0) v_{i x}(\tau) v_{i z}(\tau)>\approx<h^{2}>\left(\frac{1}{15}+\frac{8 c}{15}+\frac{2 c^{2}}{5}\right)<\left[v_{i x}^{2} v_{i z}^{2}-v_{i x}^{2} v_{i z} V_{\xi z}-v_{i z}^{2} v_{i x} V_{\xi x}\right. \\
+ & \left.v_{i x} v_{i z} V_{\xi x} V_{\xi z}\right]>+<h^{2}>\left(\frac{1}{5}-\frac{2 c}{15}+\frac{c^{2}}{15}-\frac{s^{2}}{3}\right)<\left[v_{i x}^{2} v_{i z}^{2}-v_{i x}^{2} v_{i z} V_{\xi z}-v_{i z}^{2} v_{i x} V_{\xi x}\right. \\
+ & \left.v_{i x} v_{i z} V_{\xi x} V_{\xi z}\right]>+<h>\left(\frac{1}{3}+\frac{2 c}{3}\right)<\left[v_{i x}^{2} v_{i z} V_{\xi z}-v_{i x} v_{i z} V_{\xi x} V_{\xi z}\right]> \\
+ & <h>\left(\frac{1}{3}+\frac{2 c}{3}\right)<\left[v_{i z}^{2} v_{i x} V_{\xi x}-v_{i x} v_{i z} V_{\xi x} V_{\xi z}\right]>+<v_{i x} v_{i z} V_{\xi x} V_{\xi z}>
\end{aligned}
$$

where we have also used the approximation $\left\langle h^{2}[\ldots]>\approx<h^{2}><[\ldots]>\right.$ and $\left.\left.\left.<h[\ldots]\right\rangle \approx<h\right\rangle<[\ldots]\right\rangle$, and dropped the zero time dependence for brevity.

In this calculation, we note that, using the equilibrium velocity distribution (2.7), we have

$$
<v_{i x}>=0,<v_{i z}>=0,
$$

and

$$
\begin{aligned}
<v_{i x}^{2}> & =\int_{-\infty}^{\infty} \int_{-\infty}^{\infty} \ldots \int_{-\infty}^{\infty} v_{i x}^{2}\left(\frac{m}{2 \pi k_{B} T}\right)^{\frac{3 N}{2}} \exp \left(-\frac{m}{2 k_{B} T} \sum_{i=1}^{N} \mathbf{v}_{i}^{2}\right) d \mathbf{v}_{1} d \mathbf{v}_{2} \ldots . d \mathbf{v}_{N} \\
& =\int_{-\infty}^{\infty} v_{i x}^{2}\left(\frac{m}{2 \pi k_{B} T}\right)^{\frac{1}{2}} \exp \left(-\frac{m}{2 k_{B} T} v_{i x}^{2}\right) d v_{i x} \\
& =\frac{k_{B} T}{m} \\
& =<v_{i z}^{2}> \\
<v_{i x}^{2} v_{i z}^{2}> & =<v_{i x}^{2}><v_{i z}^{2}> \\
<v_{i x}^{2} v_{i z} v_{\xi z}> & =<v_{i x}^{2} v_{i z} \frac{\sum_{k=1}^{M} v_{k z}}{M}> \\
& =<v_{i x}^{2} v_{i z} \frac{v_{i z}}{M}>+(M-1)<v_{i x}^{2} v_{i z} v_{k z}>,(k \neq i) \\
& =\frac{\left\langle v_{i x}^{2} v_{i z}^{2}>\right.}{M}+(M-1)<v_{i x}^{2}><v_{i z}><v_{k z}> \\
& =\frac{<v_{i x}^{2} v_{i z}^{2}>}{M}+0
\end{aligned}
$$

and so on.

Thus, since $m=1$, the $N$ diagonal contributions of $C_{1}$ have the value

$$
<v_{i x}(0) v_{i z}(0) v_{i x}(\tau) v_{i z}(\tau)>\approx \frac{1}{M}<v_{i x}^{2} v_{i z}^{2}>\Upsilon_{c}=\left(k_{B} T\right)^{2} \Upsilon_{c}
$$


where

$$
\Upsilon_{c}=\frac{1}{3}\left[<h^{2}>\left(\frac{2}{5}+\frac{6 c}{5}+\frac{7 c^{2}}{5}-s^{2}\right)\left(1-\frac{1}{M}\right)^{2}+<h>(1+2 c)\left(\frac{2}{M}-\frac{2}{M^{2}}\right)+\frac{3}{M^{2}}\right]
$$

After similarly lengthy calculations, the $N(M-1)$ off-diagonal contributions of $C_{1}$ can be written as

$$
<v_{i x}(0) v_{i z}(0) v_{j x}(\tau) v_{j z}(\tau)>\approx\left(k_{B} T\right)^{2} \eta_{c}
$$

where

$$
\eta_{c}=\frac{1}{M^{2}}\left[<h^{2}>\left(\frac{2}{15}+\frac{2 c}{5}+\frac{7 c^{2}}{15}-\frac{s^{2}}{3}\right)-2<h>\left(\frac{1}{3}+\frac{2 c}{3}\right)+1\right]
$$

Thus,

$$
C_{1} \approx N\left(k_{B} T\right)^{2}\left[\Upsilon_{c}+(M-1) \eta_{c}\right]
$$

From [28]," The behavior over long-time intervals can be analyzed in a similar fashion. Consider $C_{2}$. Following the arguments of the last paragraph, there is a diagonal contribution proportion to $\Upsilon_{c}^{2}$ and an off diagonal contribution proportional to $2 \Upsilon_{c} \eta_{c}$, since at each time step, $M-1$ particles become correlated with particle $i$, and particle $j$ can become correlated with particle $i$ at either of the two time steps. Note, however, that there are now additional-higher order-contributions which arise, for example, when particle $j$ becomes correlated with particle $k$ which then becomes correlated with particle $i$. It is easy to see that these contributions carry additional factors of $\frac{1}{M}$ and are thus of higher order than the diagonal and direct off- diagonal contributions considered above. However, these higher off-contributions can be summed in the geometric series."

It follows that

$$
\frac{C_{n}}{N\left(k_{B} T\right)^{2}} \approx\left(\Upsilon_{c}+(M-1) \eta_{c}\right)^{n} \approx \Upsilon_{c}^{n}+n(M-1) \eta_{c} \Upsilon_{c}^{n-1}+\frac{n(n-1)(M-1)^{2} \eta_{c}^{2} \Upsilon_{c}^{n-2}}{2}+\ldots
$$

Substituting (3.28) and (3.29) into (3.12) gives

$$
\begin{aligned}
\nu & \approx \frac{\tau}{N k_{B} T} \frac{C_{0}}{2}+\frac{\tau}{N k_{B} T}\left(\sum_{n=1}^{\infty} C_{n}\right) \\
& \approx \frac{\tau}{N k_{B} T} \frac{C_{0}}{2}+k_{B} T \tau\left(\frac{\Upsilon_{c}+(M-1) \eta_{c}}{1-\left[\Upsilon_{c}+(M-1) \eta_{c}\right]}\right) \\
& =\frac{\tau}{N k_{B} T} \frac{C_{0}}{2}+\frac{k_{B} T \tau}{2}\left[\frac{10}{\left(1-\frac{1}{M}\right)\left[5-<h^{2}>\left(-1+2 \cos \alpha+4 \cos ^{2} \alpha\right)\right]}-1\right]-\frac{k_{B} T \tau}{2} .(3.3)
\end{aligned}
$$

after using (3.25) and (3.27), and simplifying.

For $C_{0}$ we have [28]

$$
C_{0}=\frac{N k_{B} T\left\langle(\Delta \xi)^{2}\right\rangle}{\tau^{2}}
$$


and $\left\langle(\Delta \xi)^{2}\right\rangle \approx \frac{1}{6}+\lambda^{2}$. Since, the mean free path $\lambda=\tau \sqrt{k_{B} T}$, the viscosity now becomes

$\nu \approx \frac{\tau}{2 N k_{B} T}\left[\frac{N k_{B} T}{\tau^{2}}\left(\frac{1}{6}+\lambda^{2}\right)\right]+\frac{k_{B} T \tau}{2}\left[\frac{10}{\left(1-\frac{1}{M}\right)\left[5-<h^{2}>\left(-1+2 \cos \alpha+4 \cos ^{2} \alpha\right)\right]}-1\right]-\frac{k_{B} T \tau}{2}$.

Simplifying, the corrected shear viscosity for large mean free path is given by

$$
\nu \approx \frac{k_{B} T \tau}{2}\left[\frac{10}{\left(1-\frac{1}{M}\right)\left[5-<h^{2}>\left(-1+2 \cos \alpha+4 \cos ^{2} \alpha\right)\right]}-1\right]+\frac{1}{12 \tau} .
$$

Note that using the $x$ and $z$ components in our calculation for $C_{n}$ (see equation (3.13)) can be changed to $x$ and $y$ or $y$ and $z$ giving the same $\nu$ as obtained in (3.35). 


\section{Chapter 4}

\section{Numerical Results}

In this Chapter, we provide the numerical results for MPC flow through a cylinder with a local constriction using the MPC dynamics outlined in Chapter 2. We describe the flow domain in the first section, and the cell-energy generation from an implementation perspective thereafter. Next we provide the numerical results for MPC flow through the constriction. We determine the theoretically predicted viscosity derived in this Thesis and the theoretical estimate for viscosity without use of a force/thermostat, and compare the resulting predicted maximum centerline velocities to the numerically obtained profiles. Next, effects of varying the force, the degree of constriction, and the wall slip value are presented and discussed.

\subsection{Geometry of the cylinder}

Figure 4.1 shows a cross-section of the geometry of the cylinder, where $y$ is chosen as the coordinate in the axial direction. Assuming axial symmetry of the entire flow domain, including the constricted portion of the cylinder, we can define the flow domain through specification of a $y$-dependent radius, $R(y)$. As developed in Akther [1], we impose that the radius of the cylinder be a differentiable function of $y$ with the unconstricted portion of the cylinder having constant radius $R_{0}$. Let the constriction start at $y_{1}$, have minimum radius $R_{0}-\delta$ at $y_{2}$, and end at $y_{3}$. Assuming a third-order polynomial for $R(y)$ in the form

$$
R(y)= \begin{cases}R_{0} & \text { if } y \leq y_{1}, \\ a y^{3}+b y^{2}+c y+d=A(y) & \text { if } y_{1}<y \leq y_{2}, \\ e y^{3}+f y^{2}+g y+h=B(y) & \text { if } y_{2}<y \leq y_{3}, \\ R_{0} & \text { if } y \geq y_{3},\end{cases}
$$


the values of the eight constants $a, b, c, d, e, f, g$ and $h$ can be computed using the following conditions: Continuity of $R(y)$ requires

$$
\begin{aligned}
& A\left(y_{1}\right)=R_{0}, \\
& A\left(y_{2}\right)=B\left(y_{2}\right), \\
& B\left(y_{3}\right)=R_{0} .
\end{aligned}
$$

Differentiability implies

$$
\begin{aligned}
& A^{\prime}\left(y_{1}\right)=0, \\
& A^{\prime}\left(y_{2}\right)=B^{\prime}\left(y_{2}\right), \\
& B^{\prime}\left(y_{3}\right)=0,
\end{aligned}
$$

and having a maximum constriction at $y_{2}$ requires

$$
\begin{aligned}
A\left(y_{2}\right) & =R_{0}-\delta, \\
A^{\prime}\left(y_{2}\right) & =0 .
\end{aligned}
$$

These eight conditions allow specification of the eight constants in $R(y)$.

Letting the distance between $y_{1}$ and $y_{2}$ be $l_{1}$, and between $y_{2}$ and $y_{3}$ be $l_{2}$ (see Figure 4.1), so that

$$
\begin{aligned}
& y_{2}=y_{1}+l_{1}, \\
& y_{3}=y_{1}+l_{1}+l_{2},
\end{aligned}
$$

the equation of the cylinder can be written as:

$$
R(y)= \begin{cases}R_{0}, & \text { if } y \leq y_{1}, \\ \frac{2 \delta}{l_{1}^{3}} y^{3}-\frac{3 \delta\left(2 y_{1}+l_{1}\right)}{l_{1}^{3}} y^{2}+\frac{6 \delta y_{1}\left(y_{1}+l_{1}\right)}{l_{1}^{3}} y+\frac{2 \delta y_{1}^{3}+3 \delta y_{1}^{2} l_{1}-R_{0} l_{1}^{3}}{l^{3}}, & \text { if } y_{1}<y \leq y_{2}, \\ -\frac{2 \delta}{l_{2}^{3}} y^{3}+\frac{3 \delta\left(2 y_{1}+2 l_{1}+l_{2}\right)}{l_{2}^{3}} y^{2}-\frac{6 \delta\left(y_{1}^{2}+2 l_{1} y_{1}+l_{1}^{2}+y_{1} l_{2}+l_{1} l_{2}\right)}{l_{2}^{3}} y & \\ +\frac{3 \delta l_{1}^{2} l_{2}+3 \delta y_{1}^{2} l_{2}+6 \delta y_{1} l_{1} l_{2}+2 \delta l_{1}^{3}+2 \delta y_{1}^{3}+6 \delta y_{1}^{2} l_{1}+6 \delta y_{1} l_{1}^{2}+R_{0} l_{2}^{3}-\delta l_{2}^{3}}{l_{2}^{3}}, & \text { if } y_{2}<y \leq y_{3}, \\ R_{0}, & \text { if } y \geq y_{3},\end{cases}
$$

where $x=R(y) \cos \theta$ and $z=R(y) \sin \theta$.

\subsection{Cell Energy Generation}

As part of the thermostat step in the MPC dynamics, a random energy needs to be generated in each cell at each time step, from the Gamma Distribution (see Chapter 3). There are several methods to generate random numbers from probability distributions for simulation. 


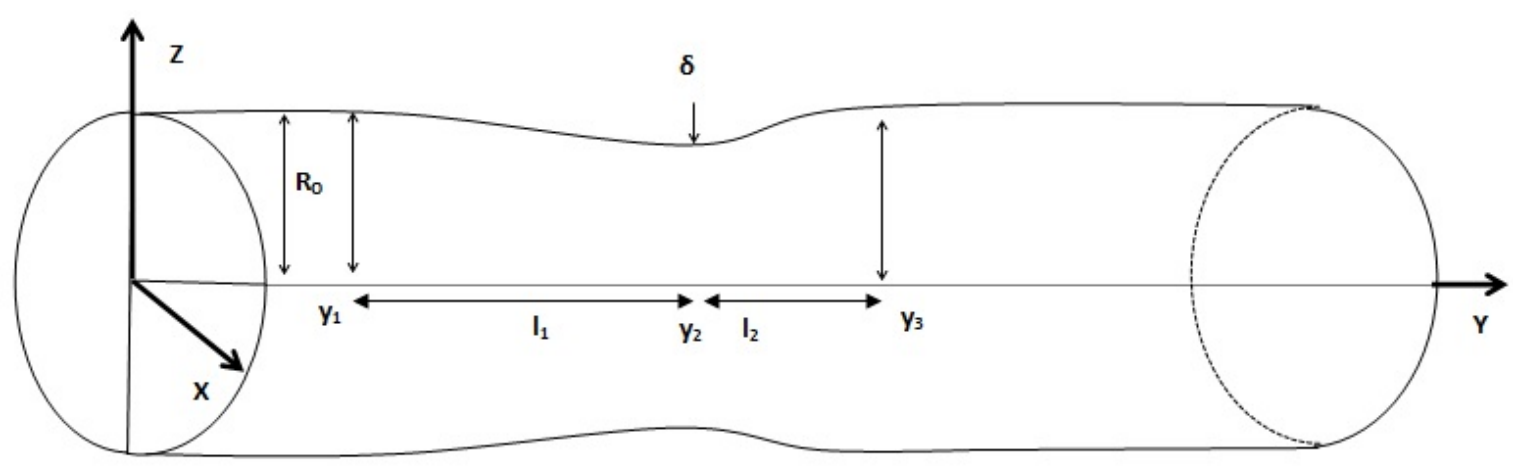

Figure 4.1: Cylinder with constriction.

\subsubsection{Acceptance Rejection Method}

The most popular and most widely applicable method for generating random numbers is the Acceptance Rejection method, which was invented by John Von Neumann [59, 19]. In this method, numbers that follow a particular distribution are generated with the help of another known distribution for which random numbers can easily be generated. The Acceptance Rejection method is particularly useful if the inverse of the desired probability distribution is not possible to determine, in which case the Inverse Transformation method to generate random numbers cannot be used. In this case, the Acceptance Rejection method is convenient and efficient for generating random numbers from discrete and continuous probability distributions.

The Acceptance Rejection method leads to random numbers from an arbitrary probability distribution function with the help of a known probability distribution function from which the process of drawing random numbers is well-known. The method is as follows. Let $F(x)$ be the desired probability distribution function and $f(x)$ the corresponding probability density function for the random variable $X$ that is generated using the Acceptance Rejection method. Let $G(x)$ and $g(x)$ respectively be the known probability distribution function and probability density function of the random variable $Y$ that will be used to draw random numbers. For the method, we will assume that the ratio $\frac{f(x)}{g(x)}$ is bounded by $k \geq 0$, namely $\sup _{x} \frac{f(x)}{g(x)} \leq k$. In practice, $k$ is as close to 1 as possible, and the closer $k$ is to 1 , the more efficient the algorithm. The details of the specific algorithm for continuous random variables are:

(i) Generate a random variable $Y$ from a chosen probability distribution function $G\left(x: \sigma_{g}^{2}, \mu_{g}\right)$, where $\sigma_{g}^{2}$ is the variance of the distribution and $\mu_{g}$ is the mean. Call the sample value $y$.

(ii) Generate a random variable $U$ from the uniform distribution on the interval $[0,1]$. Call the sample value $u$.

(iii) If $u \leq \frac{f(y)}{k g(y)}, u \in[0,1]$, accept $y$ as a realization of $f(x)$ (of $X$ ), otherwise reject $u$, and repeat (i) to (iii).

Then, $Y$ is a random variable corresponding to the desired probability density function $f(y)$. 


\subsubsection{Cell energy generation for MPC}

Using the Acceptance Rejection method, we now describe the process of generating random numbers that follow the conditional probability distribution $p\left(E_{\xi} \mid N_{\xi}\right)$ as given in (2.16). We assume that $E_{\xi} \in \mathbb{R}^{+}$ and that $N_{\xi}$ is a countable number. For simplicity we write $p(E)$ instead of $p\left(E_{\xi} \mid N_{\xi}\right)$ and we omit the subscript $\xi$ in $E$ and $N$.

For the probability density function in (i), as in [23], we choose for convenience the exponential distribution

$$
g(E)=\frac{\alpha}{c} \exp \left(-\alpha\left|E-E_{m}\right|\right)
$$

where $c=2-\exp \left(-\alpha E_{m}\right), E_{m}=\frac{\frac{\phi}{2}-1}{\beta}, \alpha=\beta \sqrt{\frac{2}{\phi}}$, and $\beta=\frac{1}{k_{B} T}$. To generate random numbers from the exponential distribution, we use the Inverse Method.

Let $G(E)$ be the cumulative probability distribution function for the random variable $E$. Then

$$
\begin{aligned}
G(E) & =\int_{0}^{E} g(x) d x, \\
& = \begin{cases}1-\frac{1}{c} e^{\alpha E_{m}} e^{-\alpha E} & \text { if } E<E_{m}, \\
\frac{1}{c} e^{-\alpha E_{m}}\left(e^{\alpha E}-1\right) & \text { if } E>E_{m} .\end{cases}
\end{aligned}
$$

By definition of a probability distribution function, $0 \leq G(E) \leq 1$. Thus, using the Inverse Method, we let $G(E)$ be a uniform random variable. Then the energy $E$ of the exponential distribution is

$$
E=\left\{\begin{array}{l}
-\frac{1}{\alpha} \log (c(1-G(E)))+E_{m}, \quad \text { if } G(E)>\frac{c-1}{c}, \\
\frac{1}{\alpha} \log \left(e^{-\alpha E_{m}}+c G(E)\right)+E_{m}, \quad \text { if } G(E) \leq \frac{c-1}{c} .
\end{array}\right.
$$

This completes step (i).

For step (ii), the uniform random variable $U$ is obtained using a standard random number generator for uniform random variables.

For step (iii), it can be shown that

$$
\frac{p(E)}{g(E)} \leq c_{\max }
$$

where

$$
\begin{aligned}
c_{\text {max }} & =\max _{E}\left(\frac{p(E)}{g(E)}\right) \\
& =\frac{\alpha}{\Gamma\left(\frac{\phi}{2}\right)} \beta^{\frac{\phi}{2}} E_{\text {max }}^{\frac{\phi}{2}-1} \exp \left(-\beta E_{\max }-\alpha\left|E_{\max }-E_{m}\right|\right),
\end{aligned}
$$

since the maximum value of $c$ occurs when $E=E_{\max }=\frac{\frac{\phi}{2}-1}{\beta-\alpha}$.

Thus, we accept $E$ if $U \leq \frac{p(E)}{c_{\max } g(E)}$, otherwise we reject $E$ and repeat steps (i)-(iii). 


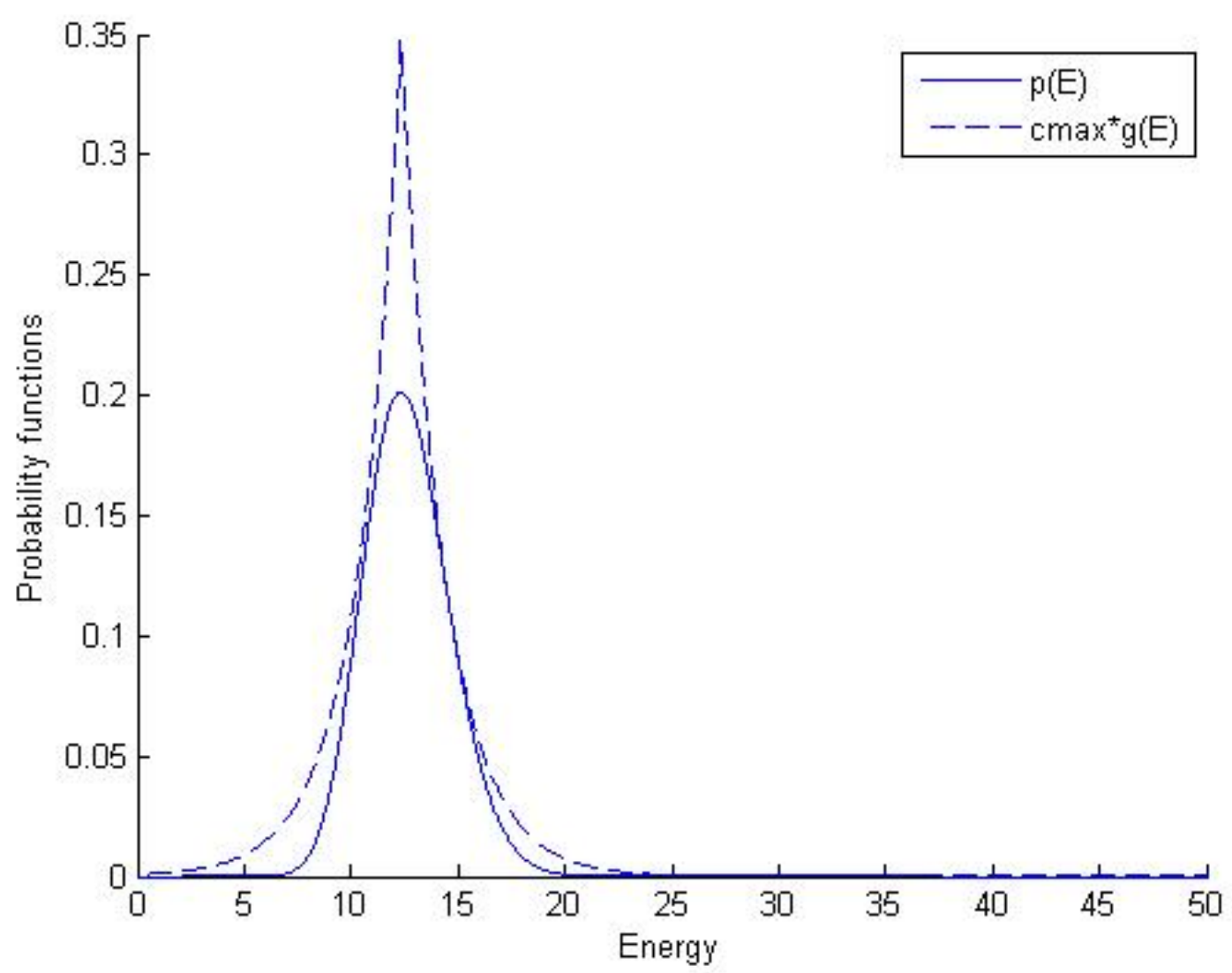

Figure 4.2: Target probability density function $p(E)$ compared to the bounding probability density function $c_{\max } g(E)$

In Figure 4.2, we plot the desired probability density function $p(E ; \beta, \phi)$ together with the exponential density function $g(E ; \alpha, \phi)$. Here $\alpha=\beta \sqrt{\frac{2}{\phi}}$ and $p(E ; \beta, \phi)<c_{\max } g(E ; \alpha, \phi)$ for all $E$, as shown in the figure. For the method to work, $p(E)$ must be bounded above by $c_{\max } g(E)$, and for the method to work most efficiently, $p(E)$ is as close as possible to $c_{\max } g(E)$.

Implementation of the Acceptance Rejection method for generating random numbers $E$ that follow the desired $p(E)$ using the above exponential density function leads to the following. In Figure 4.3 we compare the histogram of 10,000 random numbers for $E$ using the above approach, to the desired probability distribution $p(E)$. As can be seen in the Figure, there is excellent agreement between the histogram and the desired $p(E)$. 


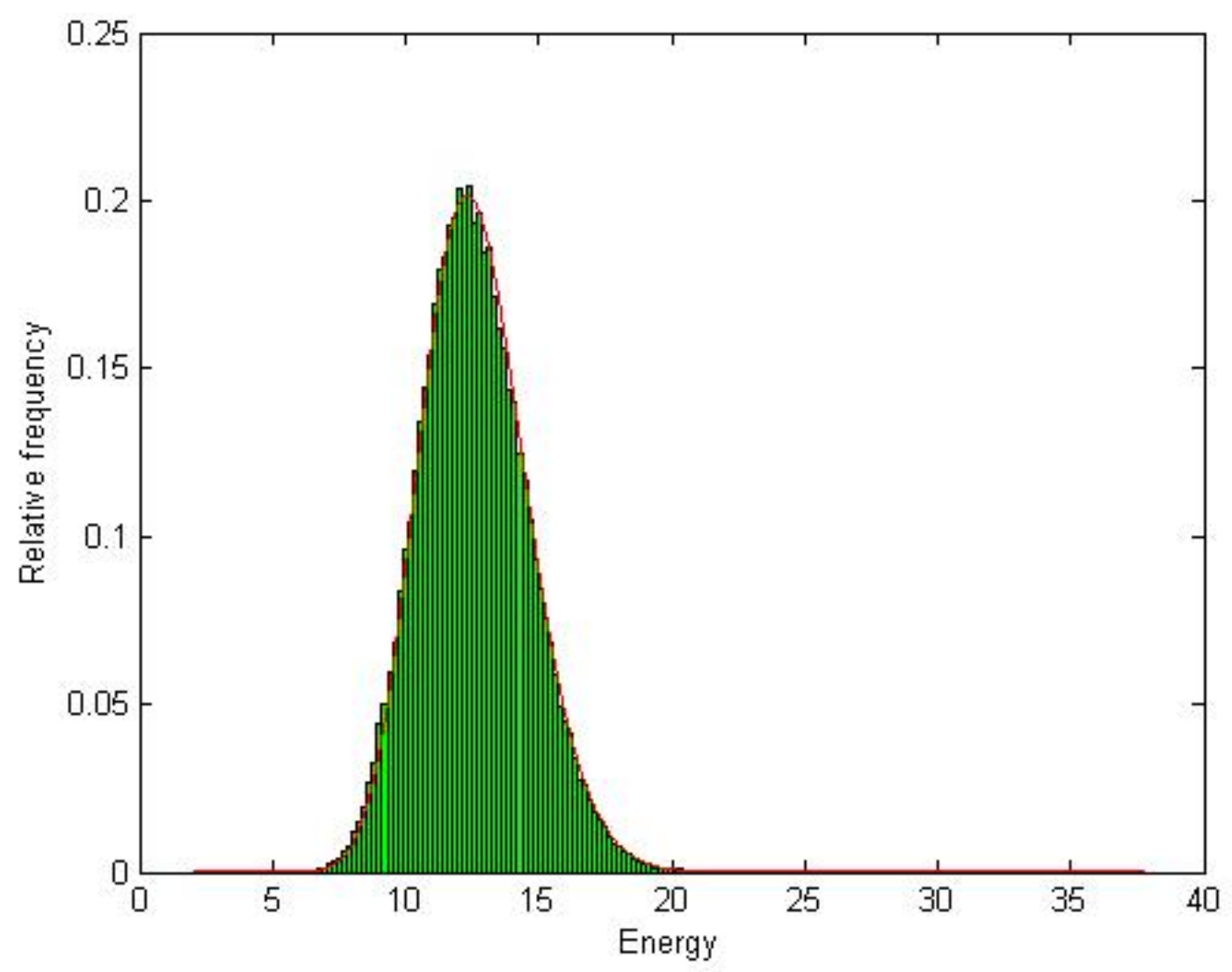

Figure 4.3: Comparison of the histogram of 10,000 random numbers generated using the Acceptance Rejection method to the target probability density curve $p(E)$. 


\subsection{Numerical Results}

For all of our MPC simulations, we used $\rho=20, m=1, k_{B} T=1, \tau=1, a_{0}=1, N \approx 20$ million, and $\Delta x=\Delta y=\Delta z=1$. There are 1200 cells in the $y$-direction and 29 cells in the $x$ and $z$ directions in the system. We considered 40000 time steps in total, with cumulative averaging starting at 5000. For the geometry, (see Figure 4.1) the radius of the cylinder in the unconstricted portion is $R_{0}=10.5$, the center of the cylinder in the $x z$ plane is $\left(x_{c}, z_{c}\right)=(14.5,14.5), l_{1}=20, l_{2}=10$, and $y_{1}=600.5$. The degree of constriction $\delta$ is varied from 0.5 to 2. Note that the value of $\delta=0$ means there is no constriction in the cylinder. The values of the force $g$ are chosen as $0.005,0.01$ and 0.02 . Increasing the value of $g$ essentially increases the flow speed in the system. For the boundary condition, values of $\lambda$ are varied from 0 to 0.5 . The value of $\lambda$ plays an important role in the boundary condition as discussed in Chapter 2. We chose the values of $\lambda=0$ (BB), $0.1,0.2$ and 0.5 (LIT) for comparison. In this thesis, the no-slip boundary condition (BB) and LIT are emphasized.

\subsubsection{Comparison of Shear Viscosity}

The function $h$ given in (2.9) is the square root of the ratio of the energy $E_{\xi}$ to kinetic energy calculated from relative velocities, namely $\left|\mathbf{v}_{i}^{\prime}-\mathbf{v}_{\xi}^{\prime}\right|^{2}$. In our theoretical estimate for the viscosity $(3.35),<h>$ is

the average value of $\sqrt{\frac{E_{\xi *\left|\mathbf{v}_{i}^{\prime}-\mathbf{v}_{\xi}^{\prime}\right|^{2}}^{2}}{2}}$ over the cells $\xi$ in the system. Using output from the simulation for approximately 3.1 million values for $h$, we estimate the value of $<h>$ and $<h^{2}>$ to be 1.0102356 and 1.0426231 respectively.

In Figure 4.4 we plot the shear viscosity with thermostat (3.35) and without thermostat (2.19). We note that using the thermostat increases the viscosity of the fluid.

In Figures 4.5 and 4.6 we plot velocity cross-sections at $y=100.5$ for MPC-BB (Fig. 4.5) and MPC-LIT (Fig. 4.6) along with the best parabolic fits using regression.

Figures 4.7 and 4.8 show the corresponding density cross-sections.

In order to determine how good our theoretical estimate for the viscosity is, we use the numerically obtained maximum centerline velocity to estimate $\nu$ for the flow. For flow through a cylinder with fixed cross-section,

$$
v(z)=\frac{m g\left(R^{2}-\left(z-z_{c}\right)^{2}\right)}{4 \nu}+v_{s}
$$

Thus, the maximum centerline velocity has the value

$$
v_{\max }=\frac{m g R^{2}}{4 \nu}+v_{s}
$$

Solving this for $\nu$ gives

$$
\nu=\frac{m g R^{2}}{4\left(v_{\max }-v_{s}\right)} .
$$




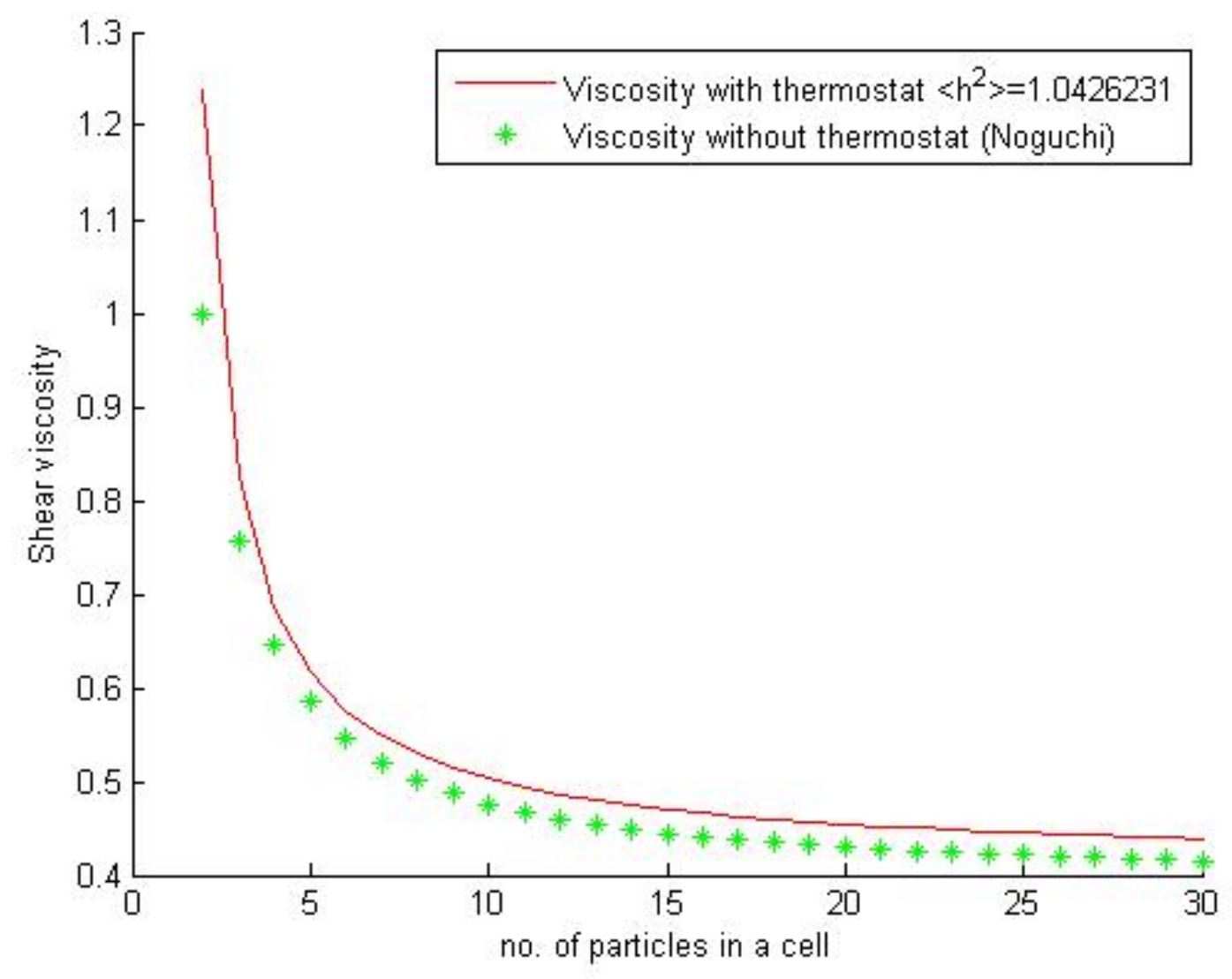

Figure 4.4: Comparison of shear viscosity with and without thermostat. 


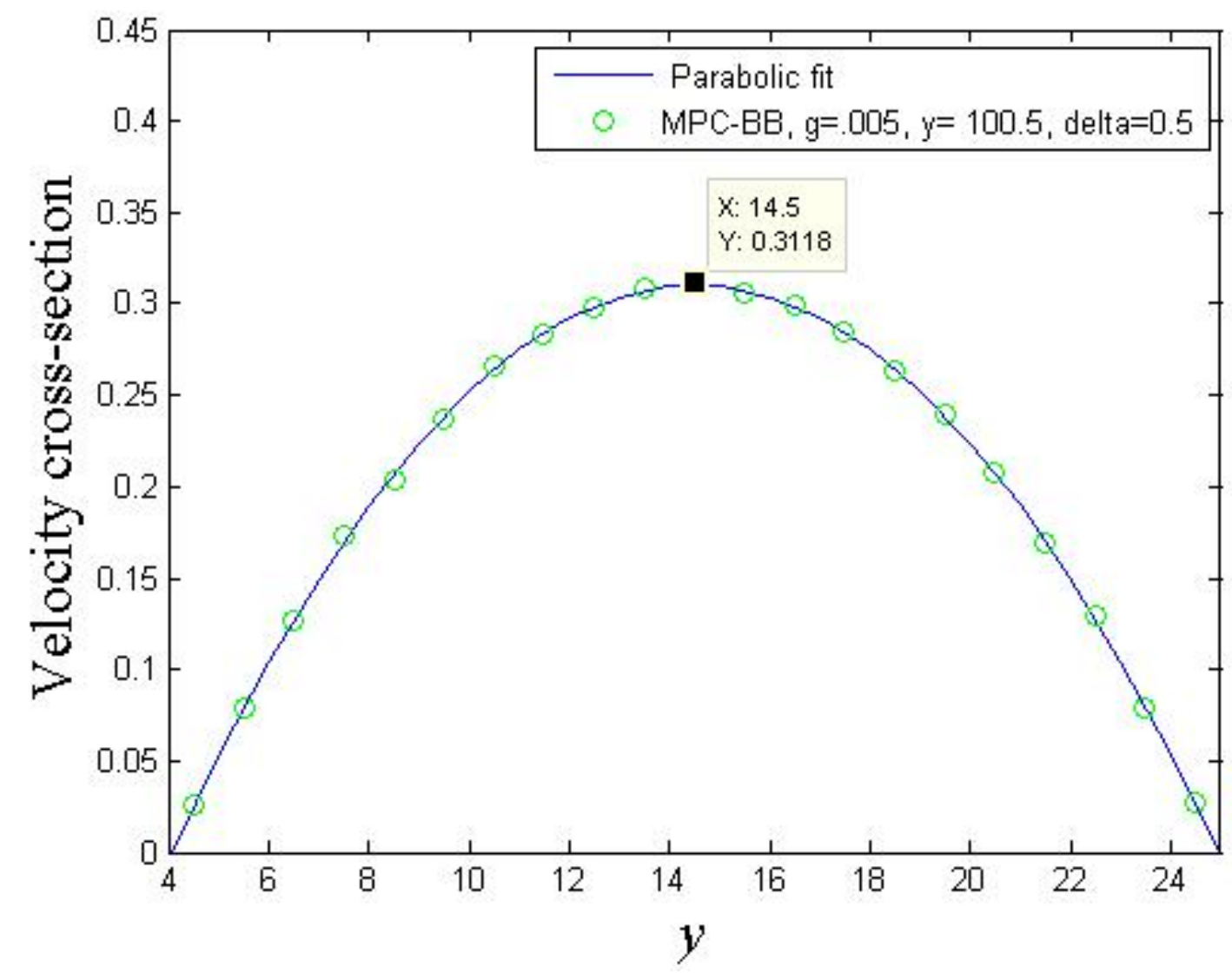

Figure 4.5: Axial velocity cross-section for MPC-BB (no-slip) with best parabolic fit. The equation for the parabolic fit is $v=-0.2885539209+0.0825548278 y-0.002845674 y^{2}$. 


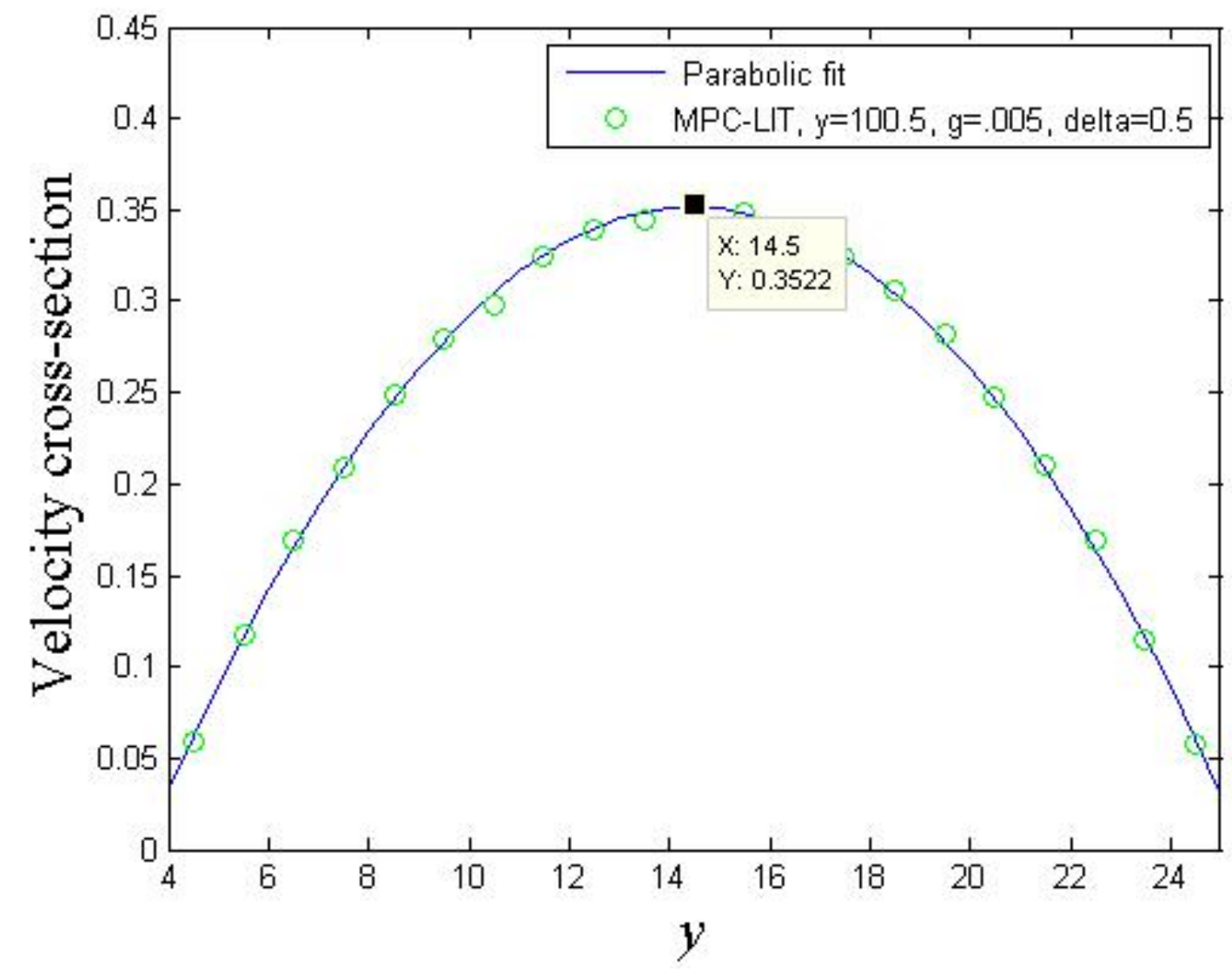

Figure 4.6: Axial velocity cross-section for MPC-LIT (slip) with best parabolic fit. The equation for the parabolic fit is $v=-0.258305808+0.084189849 y-0.002904962 y^{2}$. 


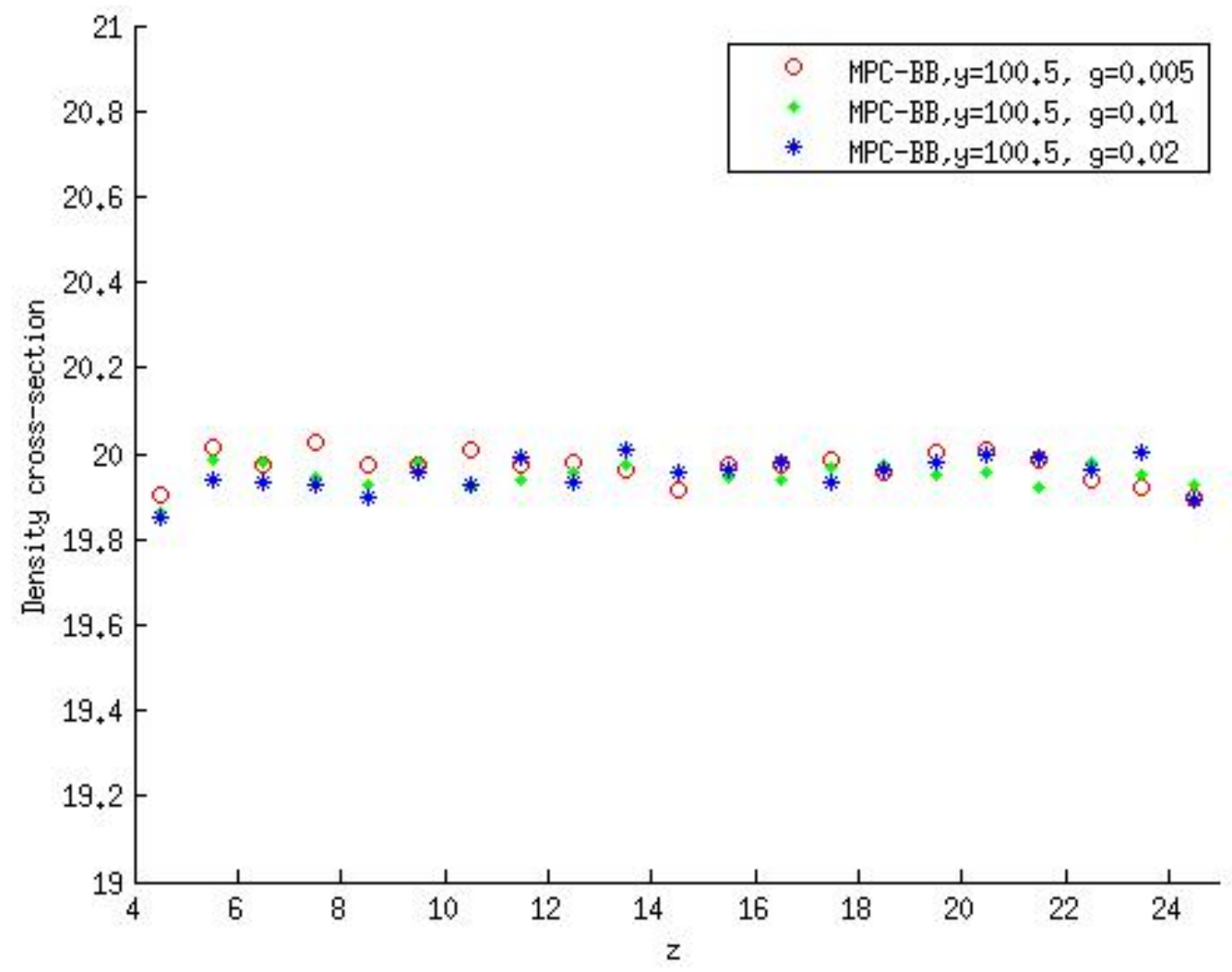

Figure 4.7: Density cross-section for MPC-BB (no-slip). 


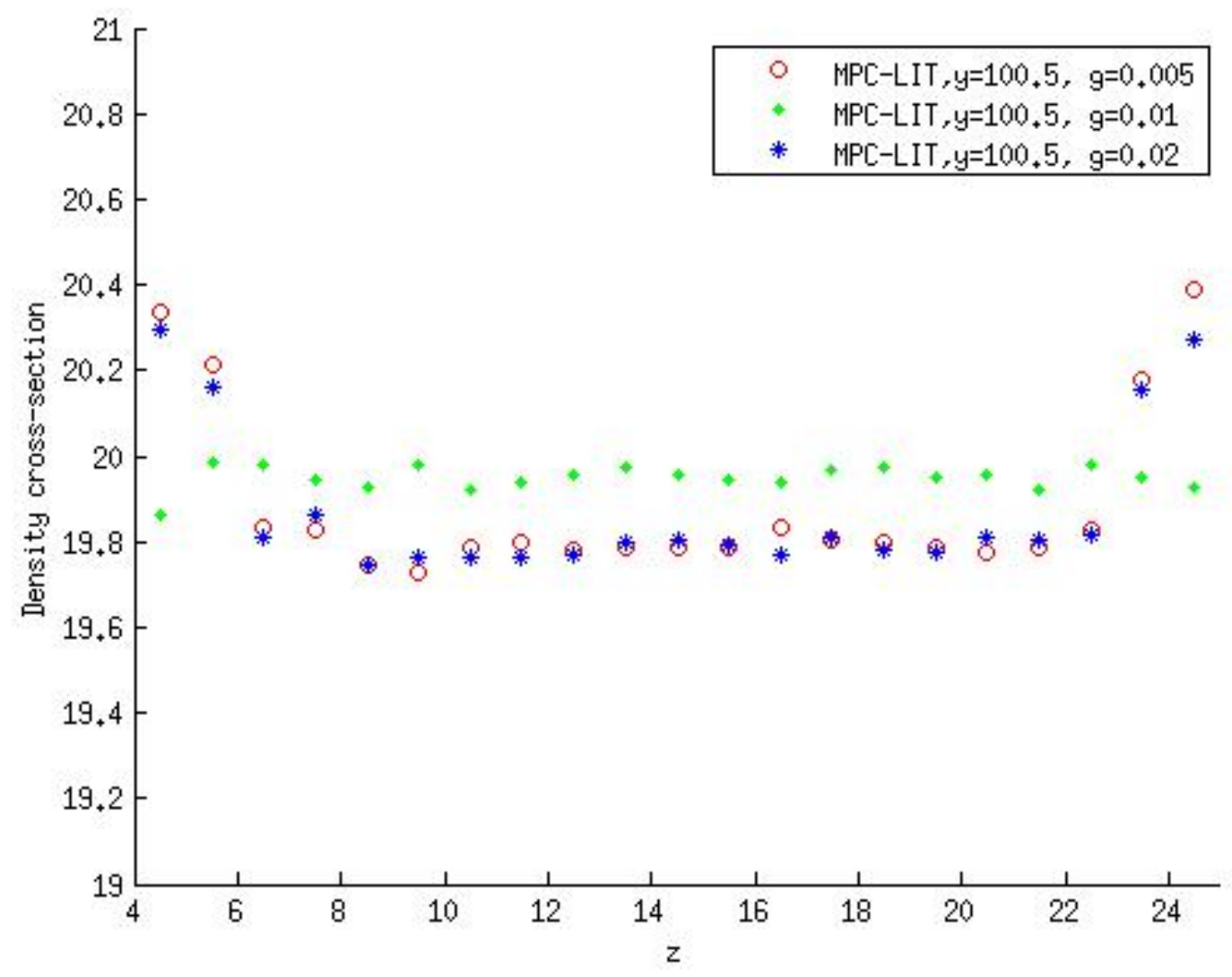

Figure 4.8: Density cross-section for MPC-LIT (slip). 


\begin{tabular}{c|c|c|c|c|c|c|c}
$\lambda$ & $v_{s}$ & $v_{\max }^{\text {num }}$ & $\nu^{\text {num }}$ & $\left\langle h^{2}\right\rangle$ & $\rho$ & $\nu^{\text {therm }}$ & $\nu^{\text {no therm }}$ \\
\hline 0.5 & 0.0320 & 0.3517 & 0.43106 & 1.1421168 & 19.88475 & 0.44091 & 0.43022 \\
0 & 0 & 0.3102 & 0.44429 & 1.0426231 & 19.96936 & 0.45441 & 0.43004
\end{tabular}

Table 4.1: Comparison of theoretically predicted viscosities to the numerically obtained value, using viscosity expressions with and without thermostat.

In Table 4.1 we compare the viscosity as predicted by (4.20) for the numerically obtained value of $v_{\max }$ (labelled $\nu^{\text {num }}$ ), to the viscosity according to our theoretically derived expression (3.35), labelled $\nu^{\text {therm }}$ and to existing expressions in the literature (labelled $\nu^{\text {no therm }}$ ).

Our theoretical expression agrees better with the numerical result in $\operatorname{LIT}(\lambda=0.5)$ than in BB $(\lambda=0)$.

\subsubsection{Effect of varying force}

In Figures 4.9 and 4.10 we plot the scaled centerline densities for various values of $g$ in a constriction with $\delta=0.5$ for both the no-slip (BB) and slip (LIT) cases. It can be seen from Figure 4.9 that in the no-slip (BB) case, the scaled centerline densities form a peak and dip as $g$ increases, with larger peaks/dips for larger $g$ values. This same trend is observed in the case of slip (LIT) in Figure 4.10. When comparing slip to no-slip, the peaks and dips are slightly larger in the case of slip, but otherwise, the curves are quite similar.

In Figures 4.11 and 4.12 we plot scaled centerline velocities for various values of $g$ in the same constriction. This time, dips are formed first (rather than peaks) that become more severe for larger values of $g$, and peaks thereafter decrease as $g$ increases. Similar trends in the case of slip (LIT) can be seen in Figure 4.12, with slightly lower dips and peaks.

Worth noting here is that in comparison to using a global thermostat (see results in [2]), the centerline density curves here lead to much larger peaks with little distinction in the qualitative behaviour of the graphs with or without slip. Likewise for the centerline velocity curves.

\subsubsection{Effect of varying degree of constriction}

In Figures 4.13 and 4.14 we plot the scaled centerline densities for various values of $\delta$ in a constriction with $g=0.005$ for both the no-slip (BB) and slip (LIT) cases. In both figures, the trend of increasing peaks/dips in the scaled centerline density curves as $\delta$ increases can be seen. There is little difference in the curves, except that the dips are slightly lower in the case of slip (see Fig. 4.14).

For the scaled centerline velocities, increasing $\delta$ has the effect of leading to a dip that is lower for larger $\delta$ values, followed by peaks that increase as $\delta$ increases (see Figure 4.15). There is very little difference in the slip and no-slip curves when comparing Figure 4.15 and Figure 4.16.

Worth noting again is the similar peaks attained in the slip and no-slip case in the scaled centerline velocities using a local thermostat (here) compared to much lower peaks in addition to post-constriction dips when using a global thermostat in [2]. 


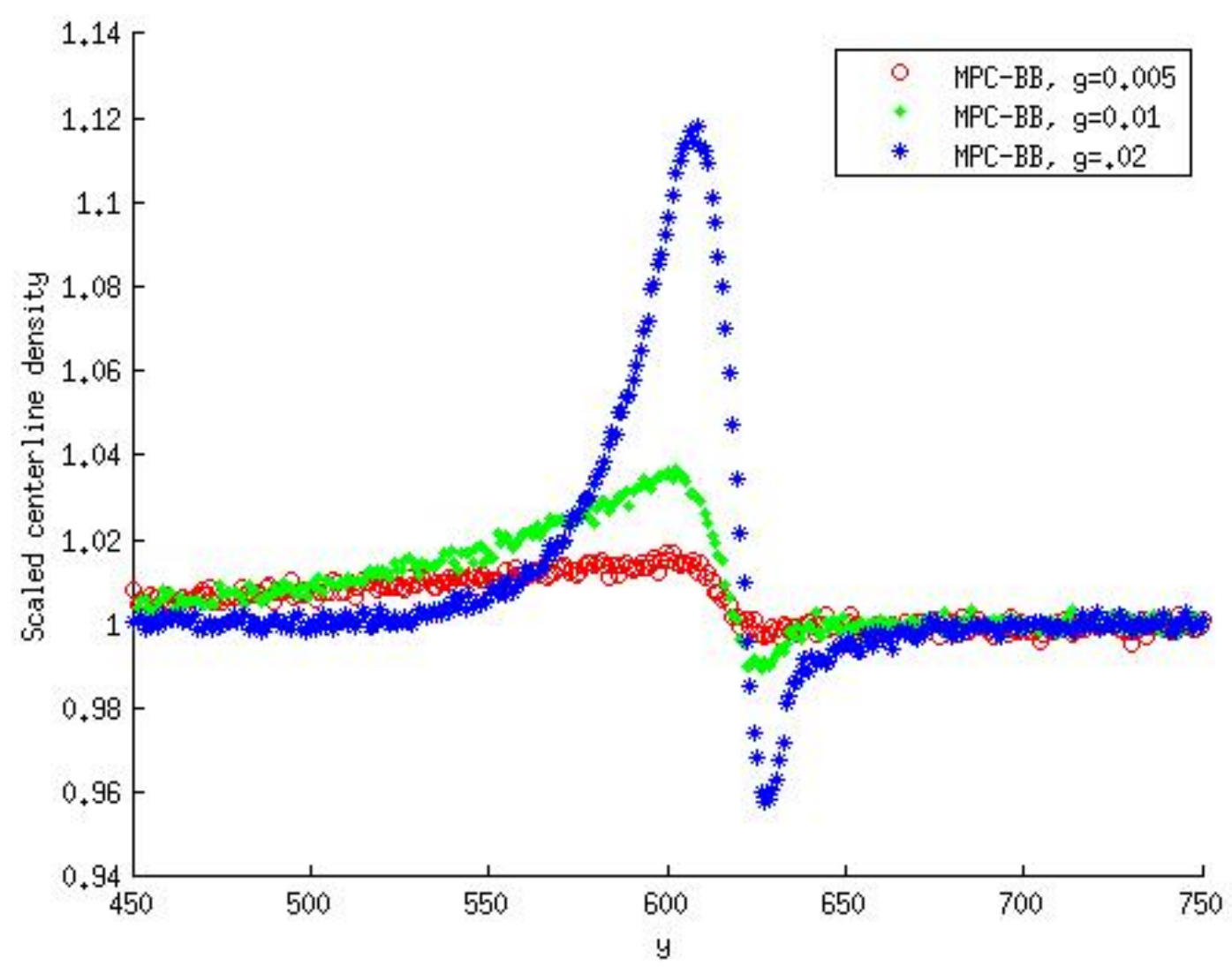

Figure 4.9: Scaled centerline densities for various values of $g$ in a constriction with $\delta=0.5$ in the no-slip $(\lambda=0, \mathrm{BB})$ case. 


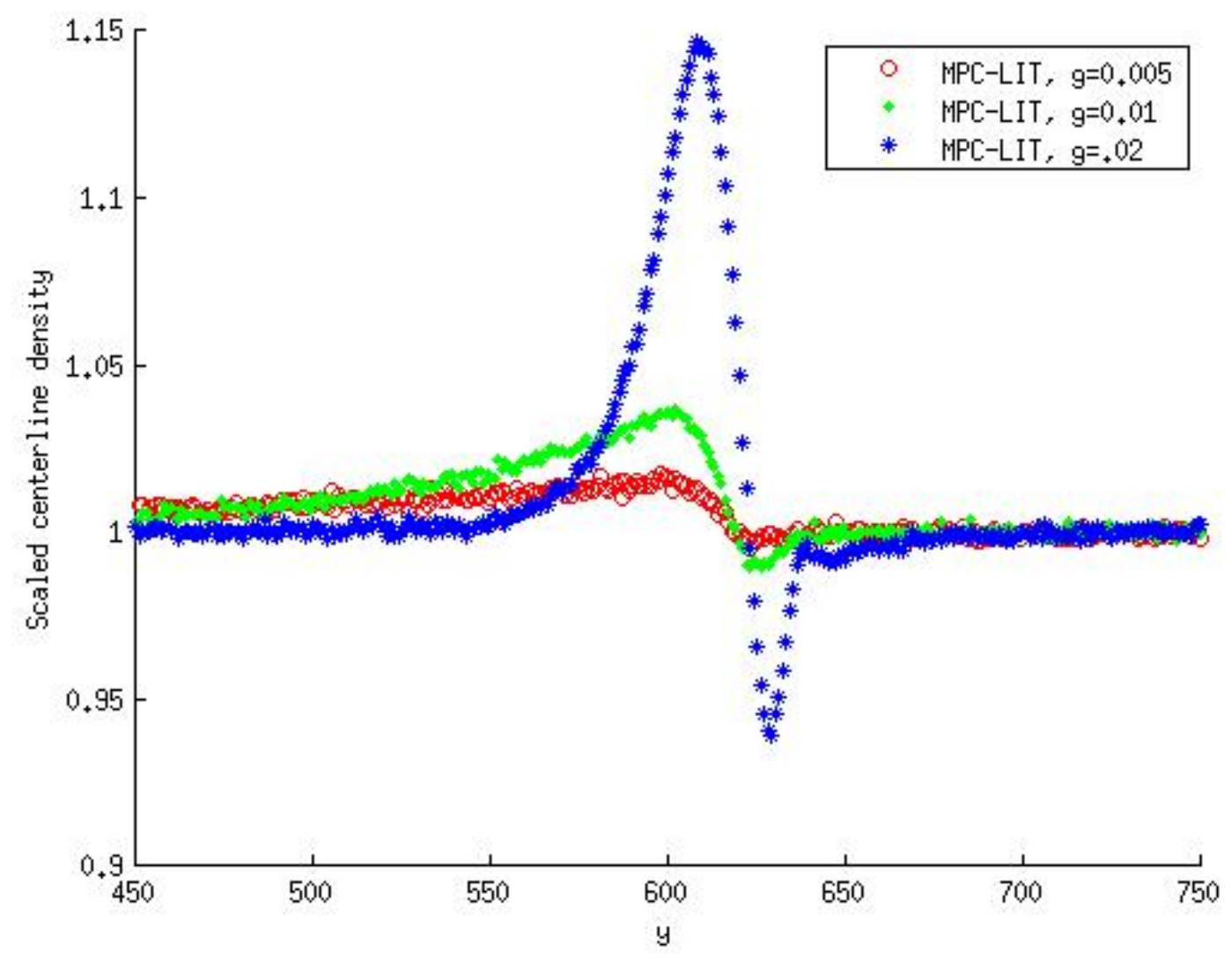

Figure 4.10: Scaled centerline densities for various values of $g$ in a constriction with $\delta=0.5$ and slip $(\lambda=0.5$, LIT $)$. 


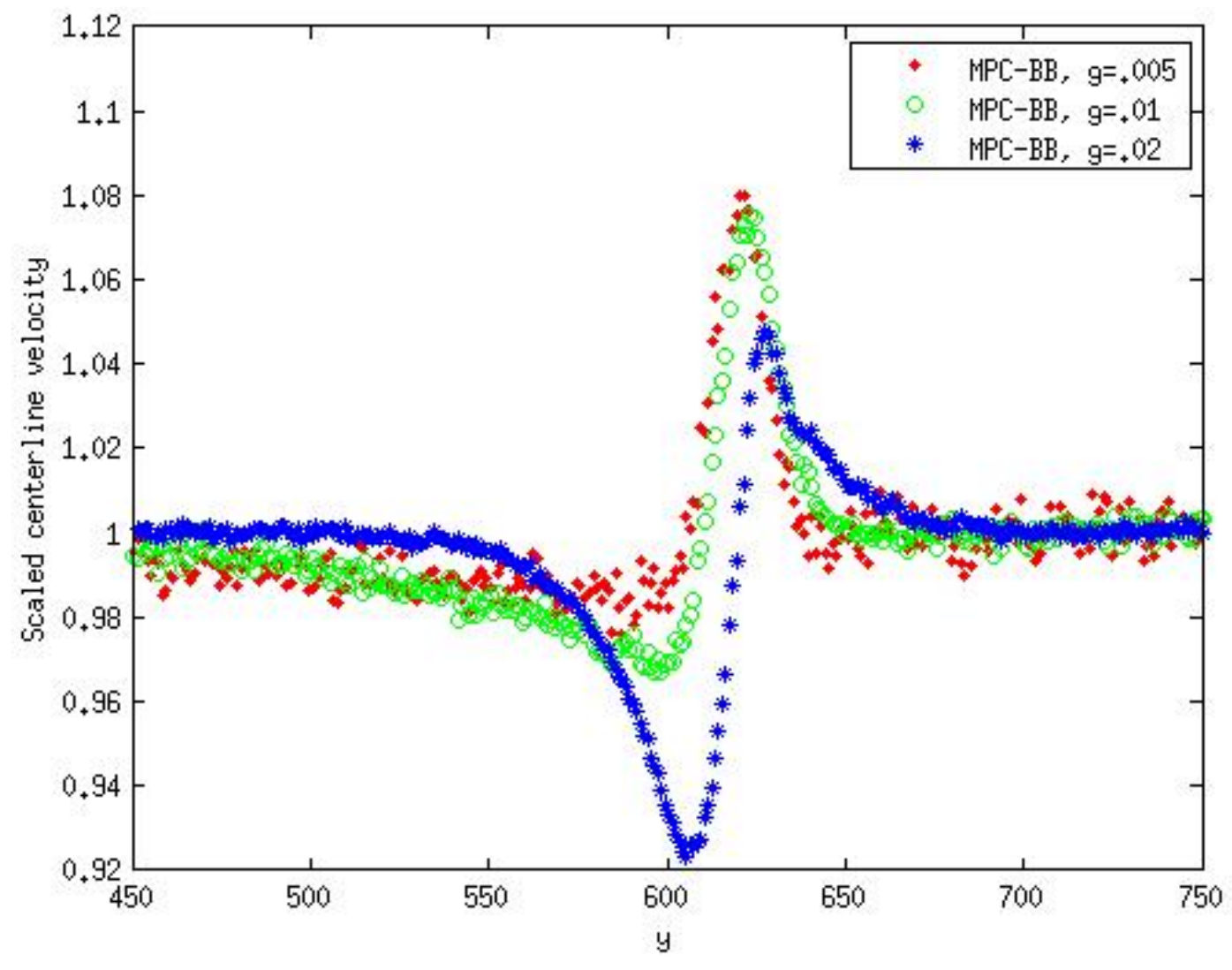

Figure 4.11: Scaled centerline velocities for various values of $g$ in a constriction with $\delta=0.5$ in the no-slip $(\lambda=0, \mathrm{BB})$ case. 


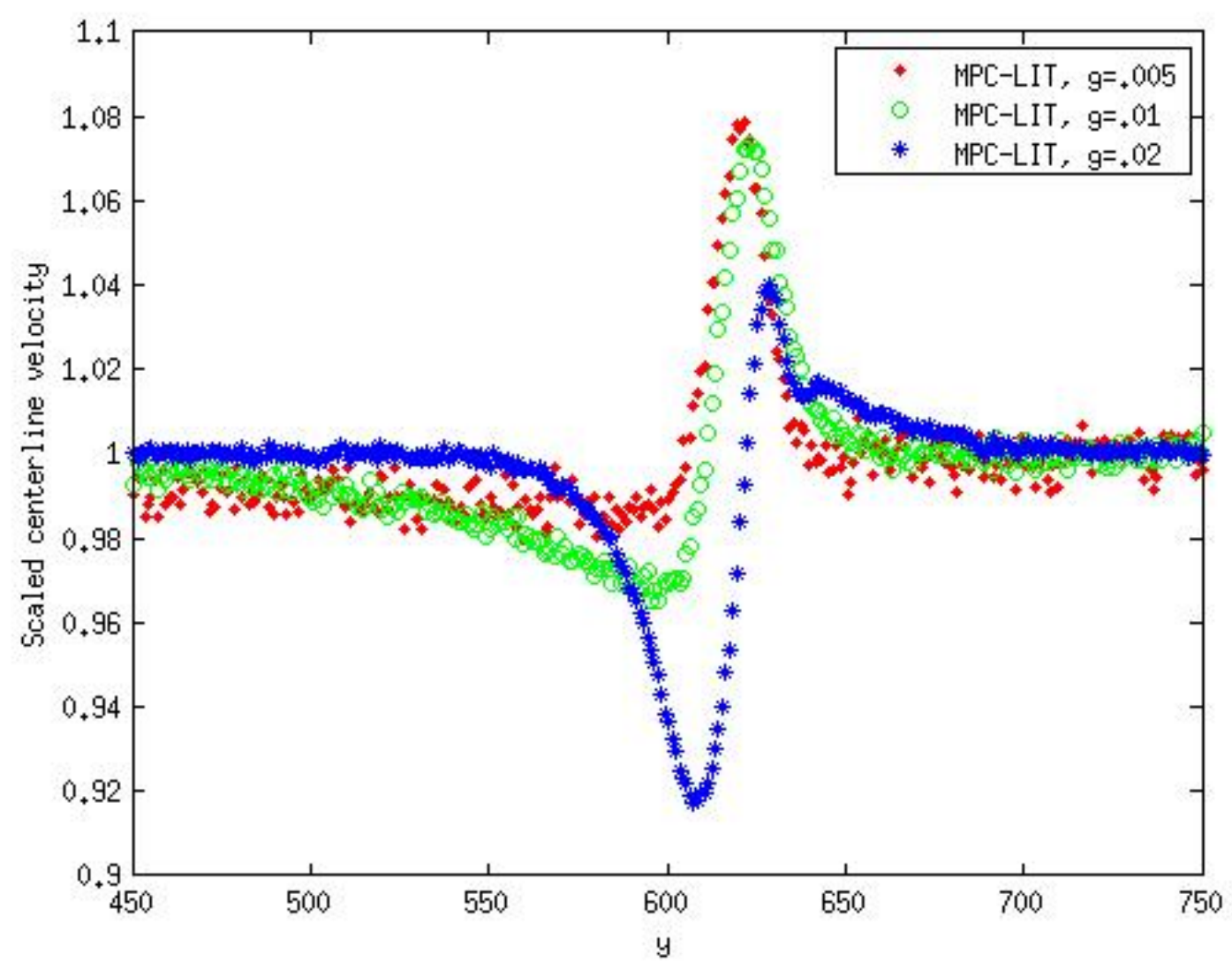

Figure 4.12: Scaled centerline velocities for various values of $g$ in a constriction with $\delta=0.5$ and slip $(\lambda=0.5, \mathrm{LIT})$. 


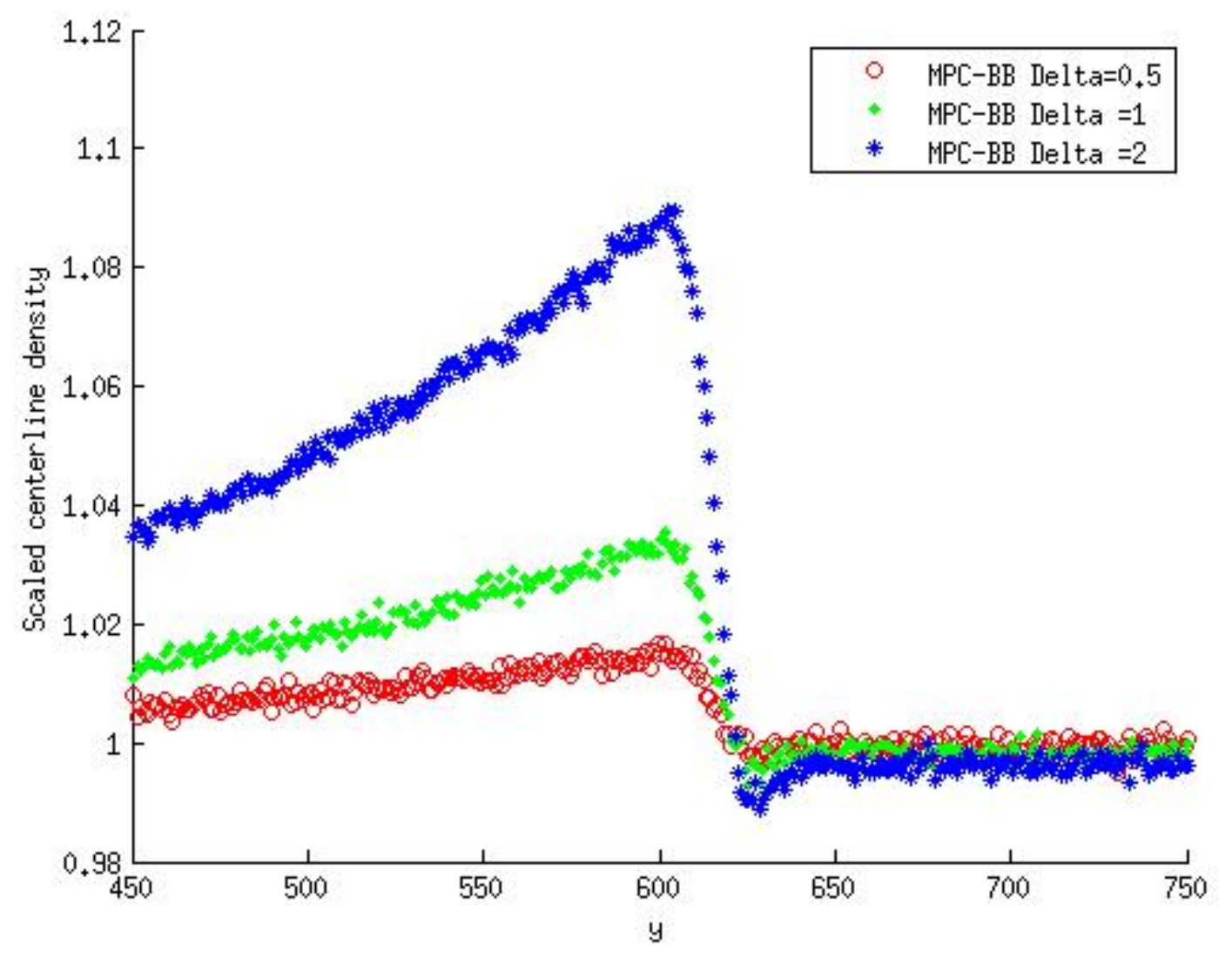

Figure 4.13: Scaled centerline densities for various values of $\delta$ in a constriction with $g=0.005$ in the no-slip $(\lambda=0, \mathrm{BB})$ case. 


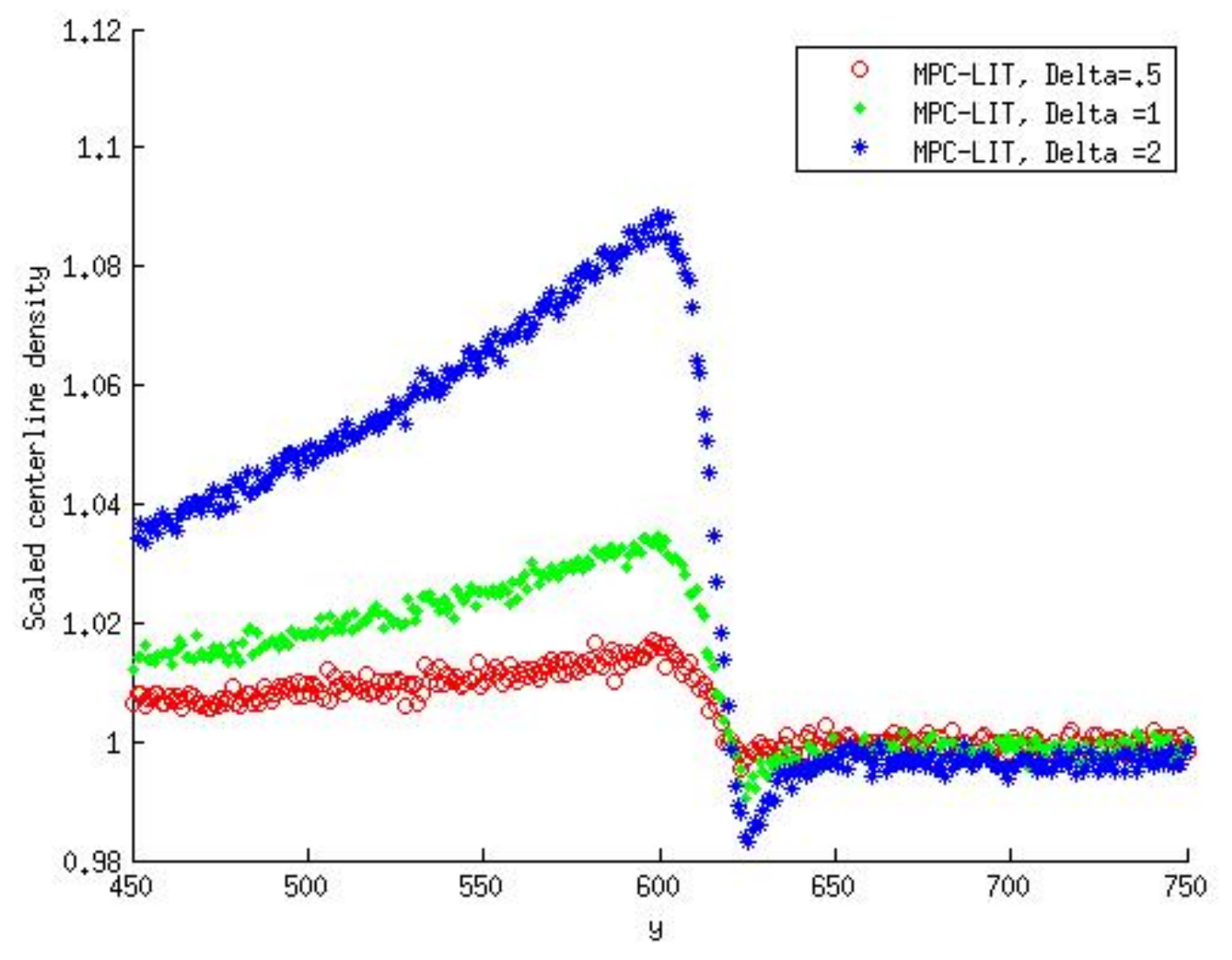

Figure 4.14: Scaled centerline densities for various values of $\delta$ in a constriction with $g=0.005$ and slip $(\lambda=0.5, \mathrm{LIT})$. 


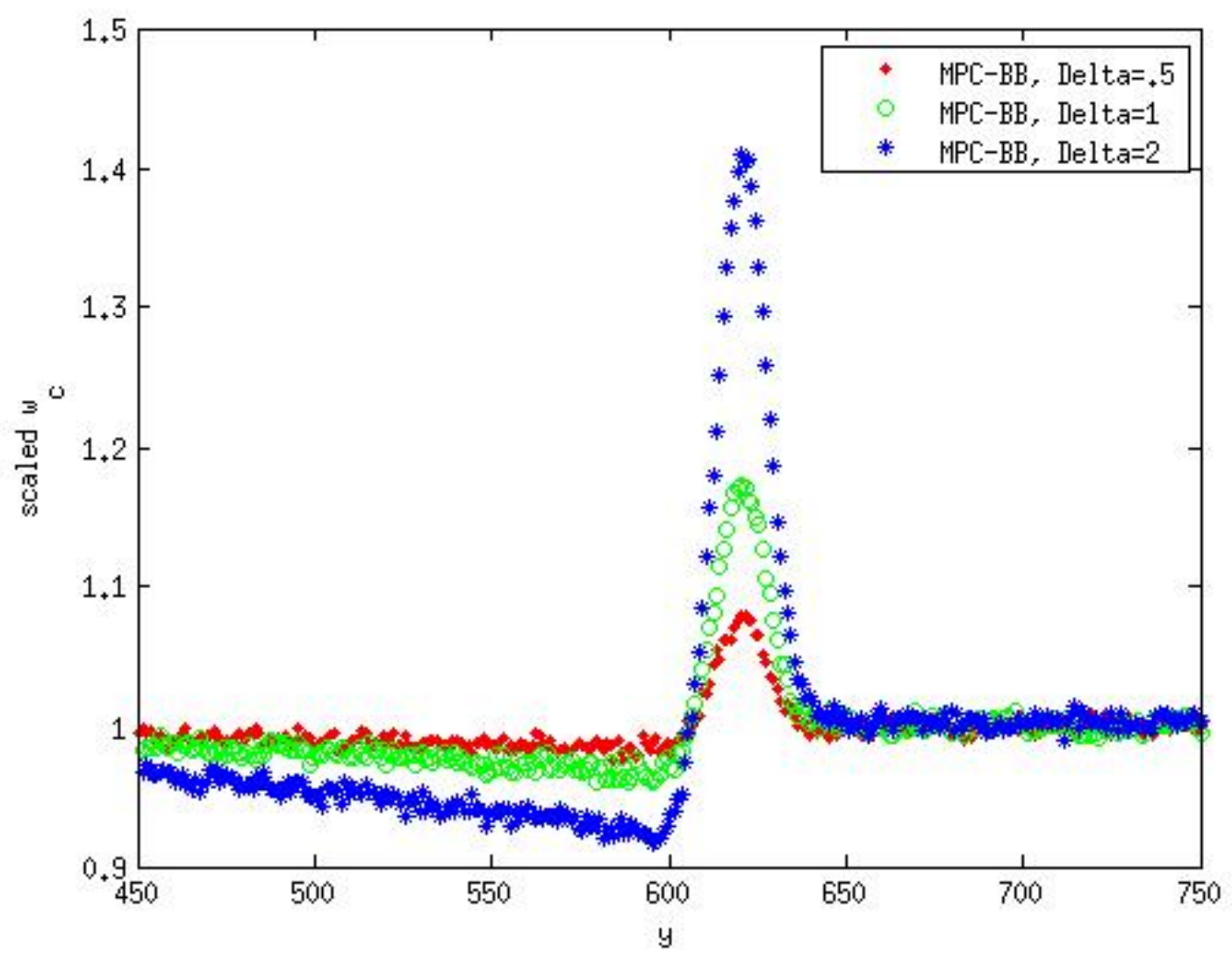

Figure 4.15: Scaled centerline velocities for various values of $\delta$ in a constriction with $g=0.005$ in the no-slip $(\lambda=0, \mathrm{BB})$ case. 


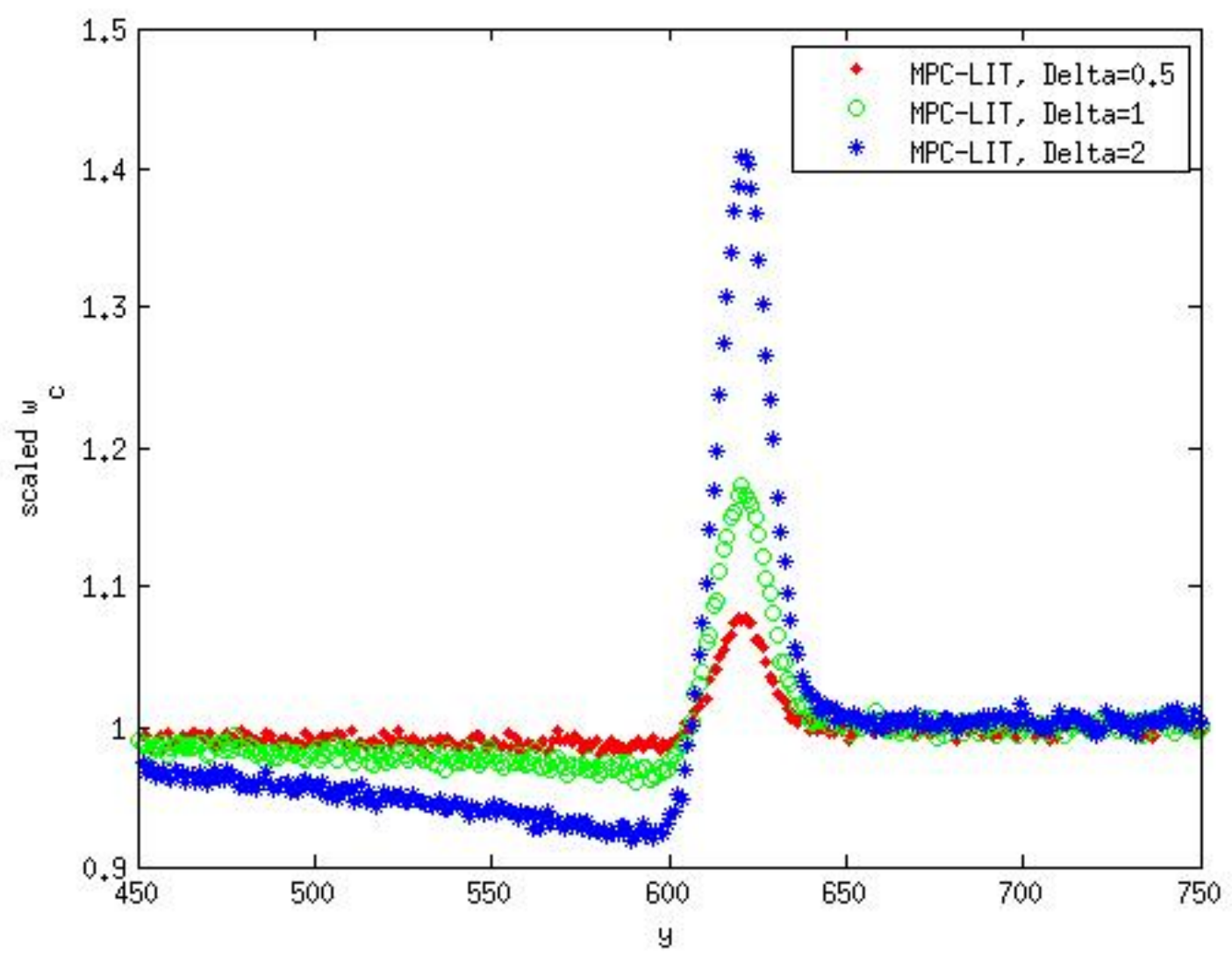

Figure 4.16: Scaled centerline velocities for various values of $\delta$ in a constriction with $g=0.005$ and slip $(\lambda=0.5, \mathrm{LIT})$. 


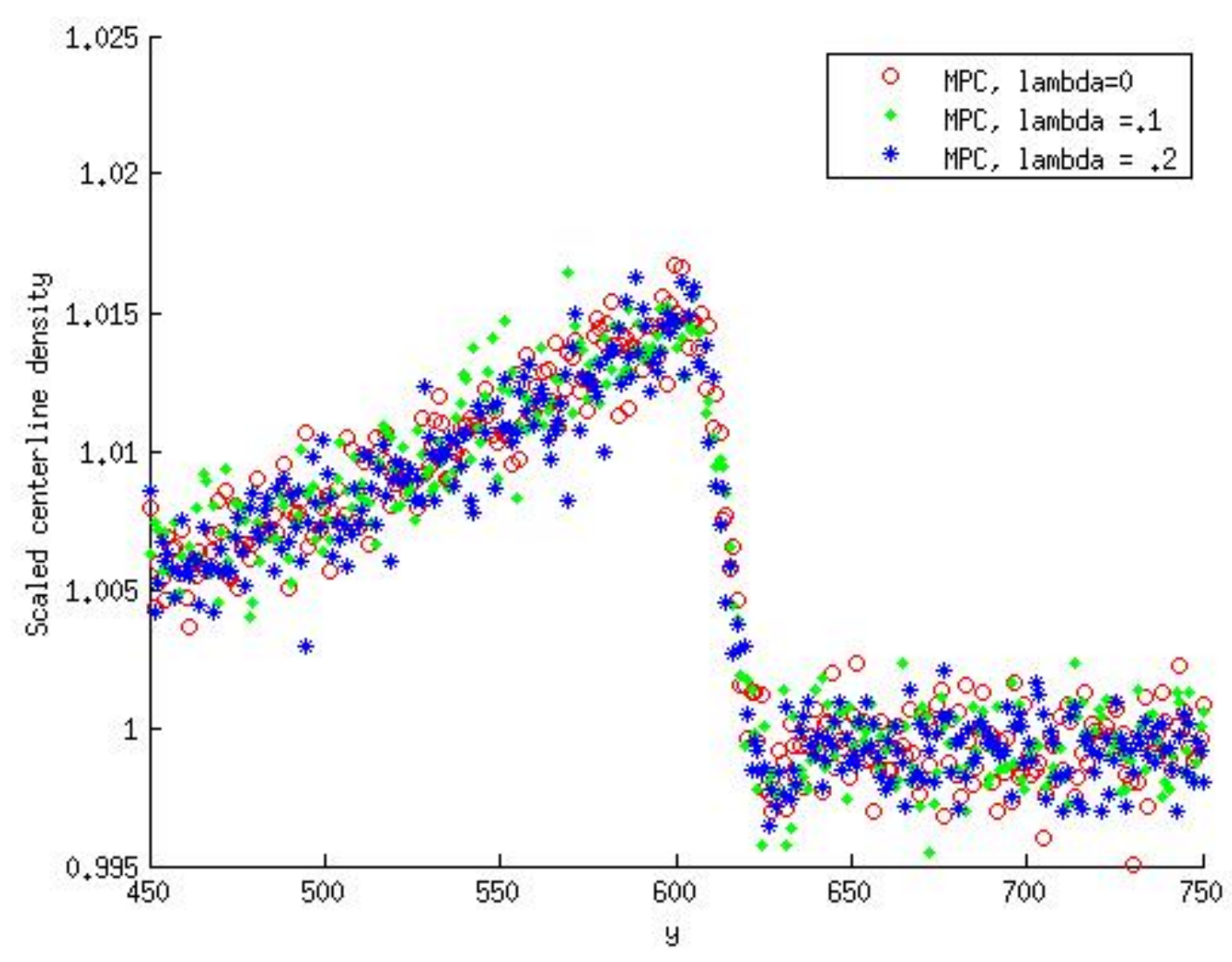

Figure 4.17: Scaled centerline densities for various values of $\lambda$ in a constriction with $g=0.005$ and $\delta=0.5$.

\subsubsection{Effect of varying slip}

In Figure 4.17 we plot the scaled centerline density in a constriction with $\delta=0.5$ and $g=0.005$, and vary slip. It can be seen that there is little change in the curve as slip increases. Similarly, there is little change in the scaled centerline velocity as slip increases as can be seen in Figure 4.18.

\subsection{Summary}

When using a local thermostat (here) compared to a global thermostat (as in [2]), the following can be observed:

(i) Adding slip leads to a parabola that is shifted upwards compared to no-slip, unlike in [2] where the maximum is lowered when slip is added. 


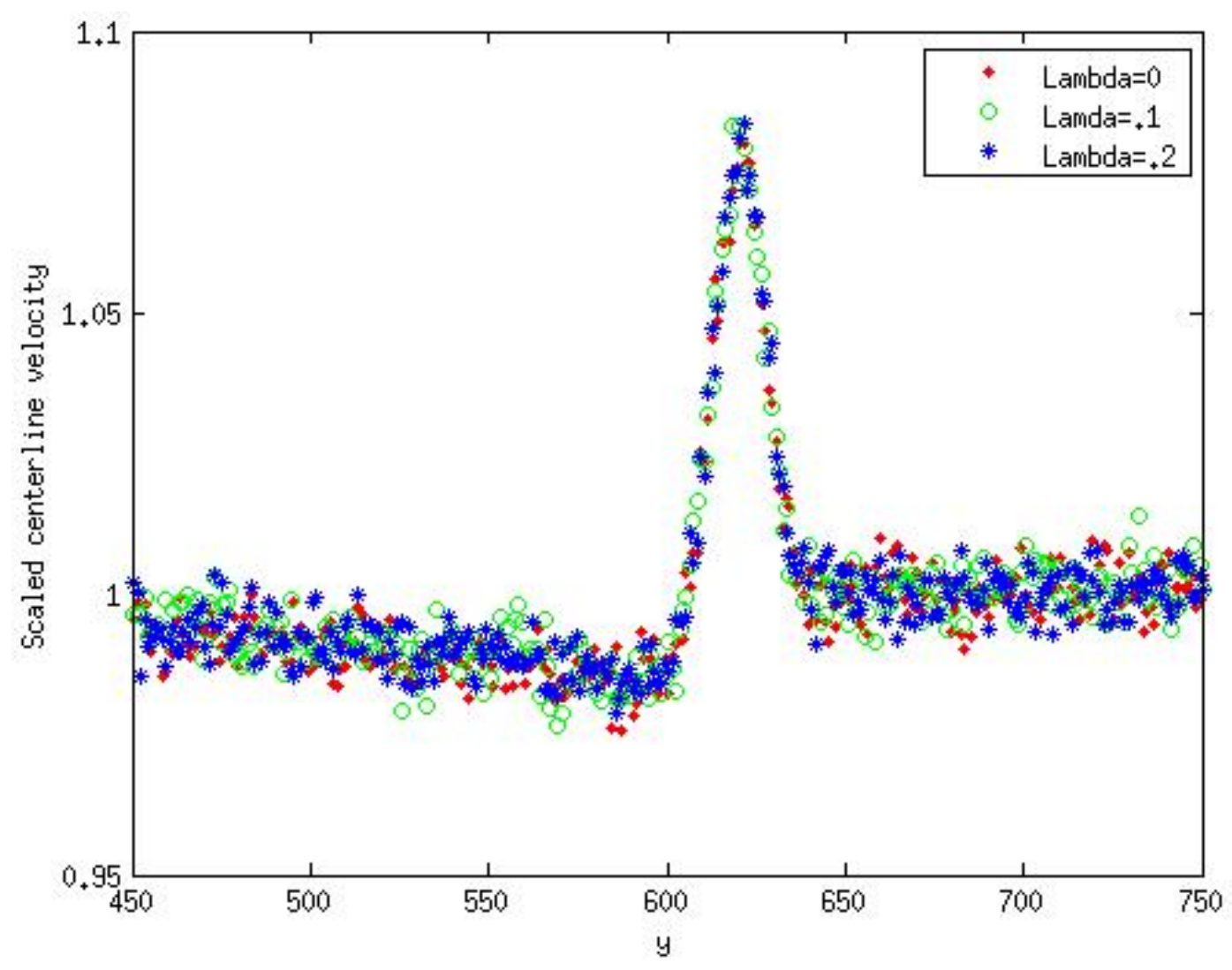

Figure 4.18: Scaled centerline velocities for various values of $\lambda$ in a constriction with $g=0.005$ and $\delta=0.5$. 
(ii) The density cross-sections in the case of slip show some accumulation of particles near the wall with a subsequent lower density in the center of the cylinder, but this effect is significantly lower with a local thermostat used here, compared to a global one used in [2]. For comparison, the density in the center here is approximately 19.8 while in [2] it is approximately 12.4 .

(iii) Scaled centerline velocity and density curves are very similar in the case of slip and no-slip, while slip curves in the case of a global thermostat had significantly different features (eg. post-constriction dip rather than pre-constriction dip).

(iv) Varying force, degree of constriction, and slip leads to expected results with use of a local thermostat, namely: increasing force or degree of constriction increases the peaks in the centerline velocity and density curves, and increasing slip seems to have little effect in a given constriction geometry.

(v) The theoretical estimate for viscosity derived in this Thesis needs further improvement in that a more theoretical estimate for $\left\langle h^{2}[\ldots]>\right.$ and $\langle h[\ldots]>$ is needed. 


\section{Chapter 5}

\section{Conclusions and Future Work}

\subsection{Conclusions}

The work in this Thesis was motivated by blood flow through the human circulatory system, which was briefly introduced in the Introduction. There have been many reports in various medical journals where it was mentioned that the viscosity of blood and that of plasma are important parameters in hemorheology. Human blood in various diseased conditions can significantly change the viscosity when compared to that of blood in healthy conditions.

In this Thesis, we considered a particle-based method called multiparticle collision (MPC) dynamics for flow simulations. In MPC dynamics, many particles in a local cell collide through an idealized rotation

operator. An external force was applied to the system in the $y$ direction to creat flow. Due to an increase in the velocities of particles, the particles gain energy and thus increase the system temperature. To maintain constant temperature, a thermostat is applied. The thermostat is a local, rather than global, thermostat, and the local energy in a cell is assumed to follow a gamma distribution. For boundary conditions, both slip (LIT) and no-slip (BB) was considered. This was discussed at length in chapter 2 of this Thesis.

In chapter 3, the shear viscosity for the MPC dynamics was derived by calculating specific correlation functions that connect to the viscosity of the system. These are called Green-Kubo relations. A large mean free path was assumed.

In chapter 4, various results for flow through a constriction were provided, in addition to viscosity estimates based on existing vicosity relations, and the one derived in this Thesis. Additionally, we noted some key feature changes in the centerline density and velocity curves when using a local thermostat (here) compared to a global thermostat that was used in [2].

Our key findings in this Thesis are as follows. In the case of no-slip, we found no significant difference in using a global versus a local thermostat in flow through a constriction. On the other hand, when slip is incorporated, scaled centerline velocity and density curves have different features when using a local rather than global thermostat. In particular, pre-constriction dips can be seen when using a local 
thermostat while a global thermostat gives rise to a post-constriction dip in the scaled centerline velocity curves. Slip and no-slip simulations give rise to similar curves, both quantitatively and qualitatively, when using a local thermostat, and this is not the case when using a global thermostat. Additionally, the velocity cross-section is found to be the no-slip parabola shifted upwards when using a local thermostat, which is quite different when a global thermostat is used. The density cross-section shows some accumulation of particles near the wall in flow with slip, but this accumulation is significantly less than when a global thermostat is used. Increasing force or degree of constriction increases the peaks in the centerline velocity and density curves, as expected, and increasing slip seems to have little effect in a given constriction geometry. Finally, our theoretical estimate for the viscosity needs some further improvement for better estimates (numerically or theoretically) for the terms involving $h$.

\subsection{Future work}

There seems to be some discrepency between the theoretical viscosity expression derived here, and that obtained numerically. Likely the approximation of $\left\langle h^{n}[\ldots]>\approx<h^{n}><[\ldots]>\right.$, for $n=1,2$ is one reason for this, and how to deal with averages involving the thermostat factor $h^{n}$ from a theoretical perspective needs further analysis.

Additionally, other flow geometries should be considered so as to test the versatility of the viscosity expression, as well as the use of the local thermostat, in MPC flow through complex flow domains. 


\section{Appendix A}

\section{Appendix}

In this appendix, we show how correlation functions relate to the Boltzmann constant.

The velocity $\mathbf{v}_{i}=\left(v_{i x}, v_{i y}, v_{i z}\right)$ in three-dimensions for particle $i$ follows the Maxwell-Boltzmann velocity distribution $(2.7)$ where $i \in[1, N]$ and $N$ is the total number of particles in the system. For particle $i,\left\langle v_{i x}^{2}(0) v_{i z}^{2}(0)\right\rangle$

$$
\begin{aligned}
= & \int_{-\infty}^{\infty} \int_{-\infty}^{\infty} \ldots \int_{-\infty}^{\infty} v_{i x}^{2}(0) v_{i z}^{2}(0)\left(\frac{m}{2 \pi k_{B} T}\right)^{\frac{3 N}{2}} \exp \left(-\frac{m}{2 k_{B} T} \sum_{i=1}^{N} \mathbf{v}_{i}^{2}\right) d \mathbf{v}_{1} d \mathbf{v}_{2} \ldots . d \mathbf{v}_{N} \\
= & \int_{-\infty}^{\infty} \int_{-\infty}^{\infty} \int_{-\infty}^{\infty} v_{i x}^{2}(0) v_{i z}^{2}(0)\left(\frac{m}{2 \pi k_{B} T}\right)^{\frac{3}{2}} \exp \left(-\frac{m}{2 k_{B} T} \mathbf{v}_{i}^{2}\right) d \mathbf{v}_{i} \prod_{j=1, j \neq i}^{N} \int_{-\infty}^{\infty} \int_{-\infty}^{\infty} \int_{-\infty}^{\infty}\left(\frac{m}{2 \pi k_{B} T}\right)^{\frac{3}{2}} \exp \left(-\frac{m}{2 k_{B} T} \mathbf{v}_{j}^{2}\right) d \mathbf{v}_{j} .
\end{aligned}
$$

Now,

$$
\begin{aligned}
& \int_{-\infty}^{\infty} \int_{-\infty}^{\infty} \int_{-\infty}^{\infty}\left(\frac{m}{2 \pi k_{B} T}\right)^{\frac{3}{2}} \exp \left(-\frac{m}{2 k_{B} T} \mathbf{v}_{j}^{2}\right) d \mathbf{v}_{j} \\
& =\left(\frac{m}{2 \pi k_{B} T}\right)^{\frac{3}{2}} \int_{-\infty}^{\infty} \int_{-\infty}^{\infty} \int_{-\infty}^{\infty} \exp \left(-\frac{m}{2 k_{B} T}\left(v_{j x}^{2}+v_{j y}^{2}+v_{j z}^{2}\right) d v_{j x} d v_{j y} d v_{j z}\right. \\
& =\left(\left(\frac{m}{2 \pi k_{B} T}\right)^{\frac{1}{2}} \int_{-\infty}^{\infty} \exp \left(-\frac{m}{2 k_{B} T} v_{j x}^{2}\right) d v_{j x}\right)\left(\left(\frac{m}{2 \pi k_{B} T}\right)^{\frac{1}{2}} \int_{-\infty}^{\infty} \exp \left(-\frac{m}{2 k_{B} T} v_{j y}^{2}\right) d v_{j y}\right) \\
& \left(\left(\frac{m}{2 \pi k_{B} T}\right)^{\frac{1}{2}} \int_{-\infty}^{\infty} \exp \left(-\frac{m}{2 k_{B} T} v_{j z}^{2}\right) d v_{j z}\right) \\
& =1 \text { because } \int_{-\infty}^{\infty} \exp \left(-a x^{2}\right) d x=\sqrt{\frac{\pi}{a}}
\end{aligned}
$$

Thus,

$$
\begin{aligned}
& \left\langle v_{i x}^{2}(0) v_{i z}^{2}(0)\right\rangle=\int_{-\infty}^{\infty} \int_{-\infty}^{\infty} \int_{-\infty}^{\infty} v_{i x}^{2}(0) v_{i z}^{2}(0)\left(\frac{m}{2 \pi k_{B} T}\right)^{\frac{3}{2}} \exp \left(-\frac{m}{2 k_{B} T} \mathbf{v}_{i}^{2}\right) d \mathbf{v}_{i} \\
& =\int_{-\infty}^{\infty} \int_{-\infty}^{\infty} \int_{-\infty}^{\infty} v_{i x}^{2}(0) v_{i z}^{2}(0)\left(\frac{m}{2 \pi k_{B} T}\right)^{\frac{3}{2}} \exp \left(-\frac{m}{2 k_{B} T}\left(v_{i x}^{2}+v_{i y}^{2}+v_{i z}^{2}\right)\right) d v_{i x} d v_{i y} d v_{i z}
\end{aligned}
$$




$$
\begin{aligned}
& =\int_{-\infty}^{\infty} \int_{-\infty}^{\infty} v_{i x}^{2}(0) v_{i z}^{2}(0) \frac{m}{2 \pi k_{B} T} \exp \left(-\frac{m}{2 k_{B} T}\left(v_{i x}^{2}+v_{i z}^{2}\right)\right) d v_{i x} d v_{i z} \\
& =\left(\frac{m}{2 \pi k_{B} T}\right)^{\frac{1}{2}} \int_{-\infty}^{\infty} v_{i x}^{2} \exp \left(-\frac{m}{2 k_{B} T} v_{i x}^{2}\right)\left(\int_{-\infty}^{\infty}\left(\frac{m}{2 \pi k_{B} T}\right)^{\frac{1}{2}} v_{i z}^{2} \exp \left(-\frac{m}{2 k_{B} T} v_{i z}^{2}\right) d v_{i z}\right) d v_{i x} \\
& =\left(\frac{k_{B} T}{m}\right)^{2} \\
& =\left(k_{B} T\right)^{2} \text { with } m=1 .
\end{aligned}
$$




\section{References}

[1] T. Akhter. Role of compressibility and slip in blood flow through a local constriction. Master's Thesis, Applied Mathematics, Ryerson University., 2012.

[2] T. Akhter. and K. Rohlf. Quantifying compressibility and slip in multiparticle collision (MPC) flow through a local Constriction Entropy, 16: 418-442, 2014.

[3] E. Allahyarov and G. Gompper. Mesoscopic solvent simulations: Multiparticle-collision dynamics of three-dimensional flow. Phys. Rev. E, 66:036702-1-9, 2002.

[4] G. Bardossy. Modelling blood flow in the arteries and veins of the systemic circulation. Ph. D. Thesis, Budapest, 2011.

[5] S. K. Bedkihal. Simulations of steady flows through cylindrical geometries with and without local constrictions by multiparticle collision dynamics. Master's Thesis, Biomedical Physics, Ryerson University., 2010.

[6] S. Bedkihal, J.C. Kumaradas and K. Rohlf. Steady flow through a constricted cylinder by multiparticle collision dynamics. Biomechanics and Modeling in Mechanobiology, 12:929-939, 2013.

[7] G.A. Bird. Molecular gas dynamics and the direct simulation of gas flows. Oxford University Press, 1994.

[8] D. Biswas. Blood flow models: A Comparative Study. Mittal, 2004.

[9] D. S. Bolintineanu, J.B. Lechman, S.J. Plimpton and G.S. Grest. No-slip boundary condition and forced flow in multiparticle collision dynamics. Physical Review E 86:066703, 2012.

[10] M. Brust, C. Schaefer, R.Doerr, L. Pan, M. Garcia, P. E. Arratia, and C. Wagner Rheology of Human Blood Plasma: Viscoelastic Versus Newtonian Behavior Universitat des Saarlandes, Germany, 2013.

[11] V. Chikkadi and M. Alam. Slip velocity and stresses in granular Poiseuille flow via event-driven simulation. Phys. Rev. E, 80:021303-1-16, 2009.

[12] I. P. Cho, M.P. Mooney, D.J. Cho. Hemerheological Disorder in Diabetes Mellitus. J. Diabetes Sci Technol, 2(6): 11301138, 2008. 
[13] J.R. Darias, M. Quiroga, E. Medina, P.J. Colmenares, and R. Paredes V. Simulation of suspensions in constricted geometries by dissipative particle dynamics. Molec. Sim., 29:443-449, 2003.

[14] M. T. Downton and H. Stark. Simulation of model microswimmer. Journal of Physics, Condensed Matter., 21:204101, 2009.

[15] D. J. Evans and G. P. Morris. Shear thickening and turbulence in simple fluids. Physical Review Letters, 56:2172-2175, 1986.

[16] J. H. Forrester. Flow through a converging-diverging tube and its implications in occlusive vascular disease. Ph. D. Thesis Ames, Iowa, Library, Iowa State University., 1968.

[17] J. H. Forrester and D. F. Young. Flow through a converging-diverging tube and its implications in occlusive vascular disease-I: Theoretical development. J.Biomechanics., 3:297-385, 1970.

[18] J. H. Forrester and D. F. Young. Flow through a converging-diverging tube and its implications in occlusive vascular disease-II: Theoretical and experimental results and their implications. J.Biomechanics., 3:307-310, 1970.

[19] G. E. Forsythe) John von Neumann, Collected Works. Vol. 5, Pergamon Press; Macmillan, New York, 1963, pp. 768-770. MR 28 \#1104.

[20] G. Gompper, T. Ihle, D.M. Kroll, and R.G. Winkler. Multi-particle collision dynamics: A particlebased mesoscale simulation approach to the hydrodynamics of complex fluids. Adv. Polym. Sci., 221:1-87, 2009.

[21] I. O. Gotze, H. Noguchi, and G. Gompper . Relevance of angular momentum conservation in mesoscale hydrodynamics simulations. Phys. Rev. E 76:046705, 2007

[22] D. Hershey and S. J. Cho. Blood flow in rigid tubes, thickness and slip velocity of plasma film at the wall. Journal of Applied Physiology., 21:27-32, 1966.

[23] H. Hijar and G. Sutmann. Hydrodynamic fluctuation in thermostatted multiparticle collision dynamics. Phys. Rev.w E, 83:046708, 2011.

[24] P. J. Hoogerbrugge and J. M. Koelman. Simulating microscopic hydrodynamic phenomena with dissipative particle dynamics. Europhysics Letters, 19:155-160, 1992.

[25] A. C. L. Hunt, W. A Timlake, W. P. Barnard, and Varley E. A theory of fluid flow in compliant tubes. Biophys J., 6:717-724, 1996.

[26] T. Ihle and D.M. Kroll. Stochastic rotation dynamics: A Galilean-invariant mesoscopic model for fluid flow. Phys. Rev. E, 63:020201-1-4, 2001.

[27] T. Ihle and D.M. Kroll. Stochastic rotation dynamics, formalism -I, Galilean-invariance, and GreenKubo relations. Phys. Rev. E, 67:066705, 2003. 
[28] T. Ihle and D.M. Kroll. Stochastic rotation dynamics. II.Transport coefficients, numerics, and long-time tails. Phys. Rev. E, 67:066706, 2003.

[29] R. Junker, J. Heinrich, H. Ulbrich, H. Schulte, R. Schonfeld, E. Kohle and G. Assmann. Relationship Between Plasma Viscosity and the Severity of Coronary Heart Disease. Arterioscler. Thromb. Vasc. Biol., 18:870-875, 1998.

[30] M. J. Kang, H-S. Ji, and S.J. Lee. In-vitro study on haemodiluted blood flow in a sinusoidal microstenosis. Proc. I Mech. E Part H: J. Engineering in Medicine, 224:17-25, 2009.

[31] R. Kapral. Multiparticle collision dynamics: Simulation of complex systems on mesoscales. Adv. Chem. Phys., 140:89-146, 2008.

[32] G. Koop, Dale J. Poirier, Justtin L. Tobias. Bayesian Econometric Method Cambridge University Press, 2007.

[33] E. A. Koopmana and C. P. Lowe. Advantages of a Lowe-Andersen thermostat in molecular dynamics simulation. Chem. Phys., 124:204103, 2006.

[34] A. Lamura and G. Gompper. Numerical study of the flow around a cylinder using multi-particle collision dynamics. Eur. Phys. J. E, 9:477-485, 2002.

[35] J. S. Lee. Numerical study of fluid flow through double bell-shaped constrictions in a tube. International Journal of Numerical Methods for Heat \& Fluid Flow, 12:258-289, 2002.

[36] A. Malevanets and R. Kapral. Mesoscopic model for solvent dynamics. J. Chem. Phys., 110:86058613, 1999.

[37] A. Malevanets and R. Kapral. Mesoscopic multiparticle collision model for fluid flows and molecular dynamics. Lect. Notes. Physics, Springer Verlarg Berlin Heidelberg., 640:116-149, 2000.

[38] D. K. Mandal, N. K. Manna, and S. Chakrabarti. Influence of primary stenosis on secondary one and vice versa in case of double stenosis. Journal of Applied Fluid Mechanics, 4:31-42, 2011.

[39] A. Medhavi. On macroscopic two-phase arterial blood flow through an overlapping stenosis. $e$ -Journal of Science 83 Technology (e-JST)., 2011.

[40] J.C. Misra and G.C. Shit. Role of slip velocity in blood flow through stenosed arteries: A nonNewtonian model. J. Mech. Med. Biol., 7:337-353, 2007.

[41] A. Nakano, Y. Sugii, M. Minamiyama, J. Seki, and H. Miimi. Velocity profiles of pulsatile blood flow in arterioles with bifurcation and confluence in rat mesentary measured by particle image velocimetry. JSME International Journal - Mechanical Systems Machine Elements and Manufacturing, 48:444-452, 2005.

[42] N. Noguchi, N. Kikuchi, and G. Gompper. Particle-based mesoscale hydrodynamic techniques. Europhys. Lett. 78:10005, 2007. 
[43] H. Noguchi and G. Gompper. Transport coefficients of off-lattice mesoscale-hydrodynamics simulation techniques. Phys. Rev. E, 78:016706-1-12, 2008.

[44] H. Noguchi, N. Kikuchi, and G. Gompper. Particle based mesoscale hydrodynamic techniques. European Physics Letters, 78:016706, 2007.

[45] Y. Nubar. Blood flow, slip, and viscometry. Biophys. J., 11:252-264, 1971.

[46] J.T. Padding and A.A. Louis. Hydrodynamic interactions and Brownian forces in colloidal suspensions: Coarse-graining over time and length scales. Phys. Rev. E, 74:031402-1-29, 2006.

[47] N. Padmanabhan. Mathematical model of arterial stenosis. Med. E Biol. Eng. E Computer, 18:281226, 1980.

[48] C. Picart, J.M. Piau, H. Gilliard, and P. Carpentier. Human blood shear yield stress and hematocrit dependence. J. Rheology, 42:1-12, 1998.

[49] R.N. Pralhad and D.H. Schultz. Modeling of arterial stenosis and its applications to blood diseases. Math. Biosciences, 190:203-220, 2004.

[50] D.A.P. Reid, H. Hildenbrandt, J.T. Padding, and C.K. Hemelrijk. Flow around fishlike shapes studies using multiparticle collision dynamics. Phys. Rev. E, 79:046313-1-7, 2009.

[51] D. A. P. Reid, H. Hildenbrandt, J. T. Padding, and C. K. Hemelrijk. Fluid dynamics of moving fish in a two-dimensional multiparticle collision dynamics model. Physical Review E, 85:021901, 2012.

[52] H. Schlichting. Boundary Layer Theory, 6th Edition, volume 45. McGraw-Hill, New York, 1968.

[53] J. S. Stroud and S. A. Berger. Numerical analysis of flow through a severely stenotic carotid artery bifurcation. Journal of Biomechanical Engineering., 124:9-20, 2002.

[54] E. Tüzel. Particle-based mesoscale modeling of flow and transport in complex fluids, University of Minnesota, 2007, 251 pages.

[55] E. Tüzel, G. Pan, T. Ihle and D. M. Kroll. Mesoscopic model for the fluctuating hydrodynamics of binary and ternary mixtures. Department of Physics, North Dakota State University.

[56] E. Tüzel, M. Strauss , T . Ihle and D. M. Kroll. Transport coefficients for stochastic rotation dynamics in three dimensions. Phys. Rev. E, 68:036701, 2003.

[57] M.W. Tysanner and A.L. Garcia. Non-equilibrium behaviour of equilibrium reservoirs in molecular simulations. Int. J. Numer. Meth. Fluids, 48:1337-1349, 2005.

[58] S.S. Varghese, S.H. Frankel, and P.F. Fischer. Direct numerical simulation of stenotic flows. Part 1. Steady flow. J. Fluid Mech., 582:253-280, 2007.

[59] J. von Neumann. Various techniques used in connection with random digits, in Monte Carlo Method. Appl. Math. Series, 12: 36-38, 1951. 
[60] F. M. White. Fluid Mechanics. McGraw-Hill, 2003.

[61] J.K. Whitmer and E. Luijten. Fluid-solid boundary conditions for multiparticle collision dynamics. J. Phys.: Condens. Matter, 22:104106-1-14, 2010.

[62] D. F. Young. Effect of a time-dependent stenosis on flow through a tube. J. Engng Ind, 90:248-254, 1968.

[63] J. G. Zhou. Axisymmetric lattice Boltzmann method. Phys. Rev. E, 78:036701-1-7, 2008. 